\title{
Tomographic Image Reconstruction for the Parallel-Slit Ring Collimator Fast Neutron Emission Tomography System
}

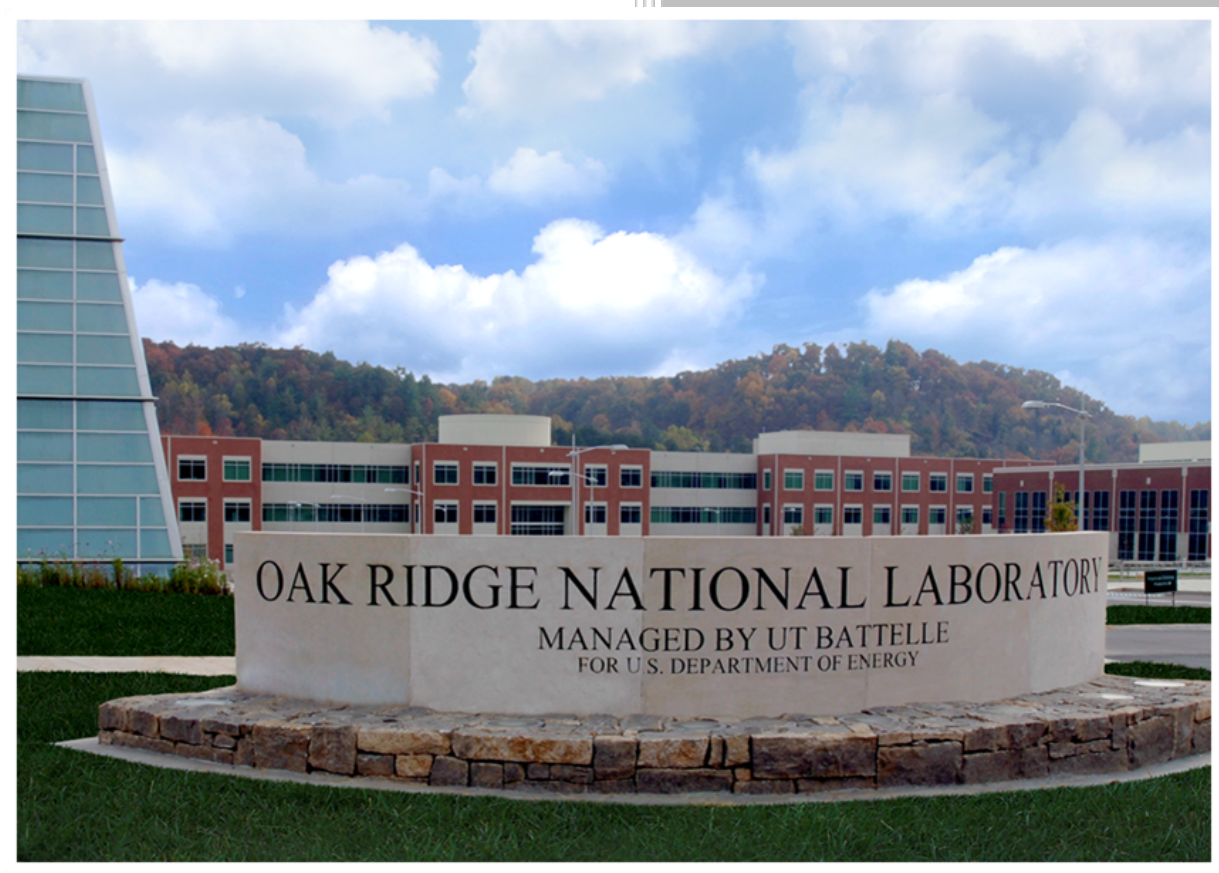

Approved for public release. Distribution is unlimited.

P. A. Hausladen

M. E. Montague

A. S. lyengar

J. Yang

April 2020 


\title{
DOCUMENT AVAILABILITY
}

Reports produced after January 1, 1996, are generally available free via US Department of Energy (DOE) SciTech Connect.

Website www.osti.gov

Reports produced before January 1, 1996, may be purchased by members of the public from the following source:

\author{
National Technical Information Service \\ 5285 Port Royal Road \\ Springfield, VA 22161 \\ Telephone 703-605-6000 (1-800-553-6847) \\ TDD 703-487-4639 \\ Fax 703-605-6900 \\ E-mail info@ntis.gov \\ Website http://classic.ntis.gov/
}

Reports are available to DOE employees, DOE contractors, Energy Technology Data Exchange representatives, and International Nuclear Information System representatives from the following source:

Office of Scientific and Technical Information

PO Box 62

Oak Ridge, TN 37831

Telephone 865-576-8401

Fax 865-576-5728

E-mail reports@osti.gov

Website http://www.osti.gov/contact.html

This report was prepared as an account of work sponsored by an agency of the United States Government. Neither the United States Government nor any agency thereof, nor any of their employees, makes any warranty, express or implied, or assumes any legal liability or responsibility for the accuracy, completeness, or usefulness of any information, apparatus, product, or process disclosed, or represents that its use would not infringe privately owned rights. Reference herein to any specific commercial product, process, or service by trade name, trademark, manufacturer, or otherwise, does not necessarily constitute or imply its endorsement, recommendation, or favoring by the United States Government or any agency thereof. The views and opinions of authors expressed herein do not necessarily state or reflect those of the United States Government or any agency thereof. 
Isotope and Fuel Cycle Technology Division

\title{
TOMOGRAPHIC IMAGE RECONSTRUCTION FOR THE PARALLEL-SLIT RING COLLIMATOR FAST NEUTRON EMISSION TOMOGRAPHY SYSTEM
}

\author{
P. A. Hausladen \\ M. E. Montague \\ A. S. Iyengar \\ J. Yang
}

April 2020

Prepared by

OAK RIDGE NATIONAL LABORATORY

Oak Ridge, TN 37831-6283

managed by

UT-BATTELLE, LLC

for the

US DEPARTMENT OF ENERGY

under contract DE-AC05-00OR22725 



\section{CONTENTS}

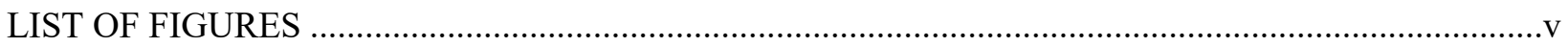

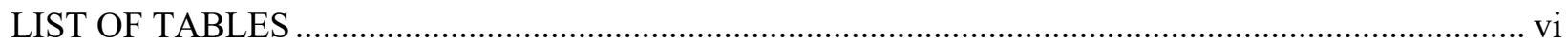

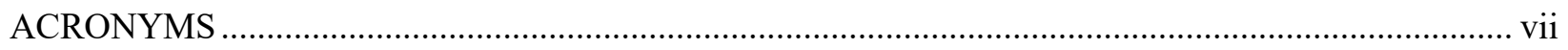

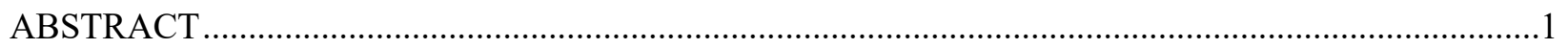

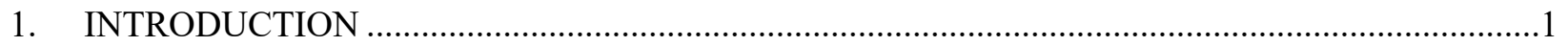

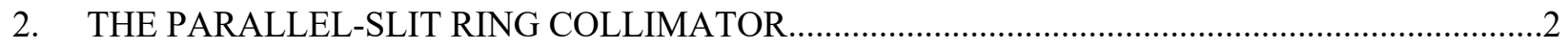

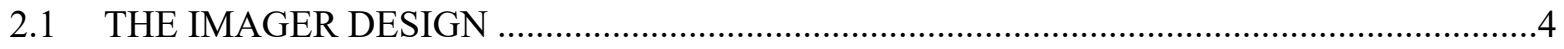

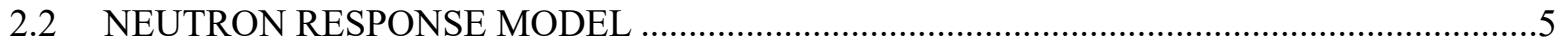

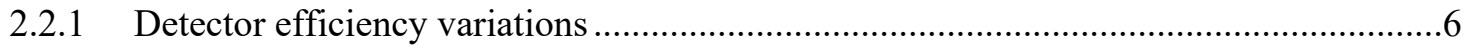

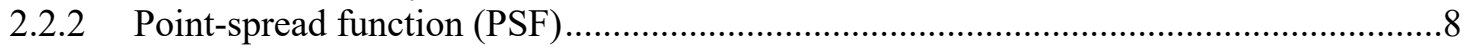

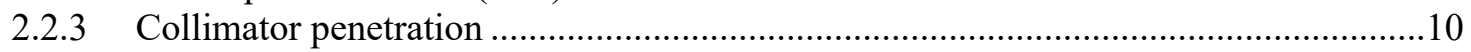

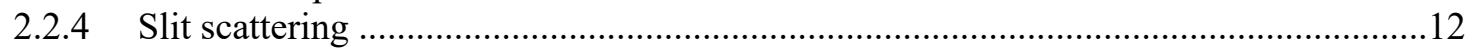

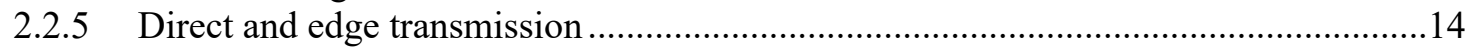

2.2.6 Comparison of the neutron response model to simulation...........................................16

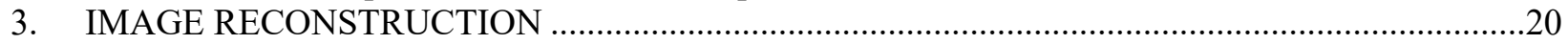

3.1 RECONSTRUCTION OF SIMULATED MEASUREMENTS OF FIVE LINE

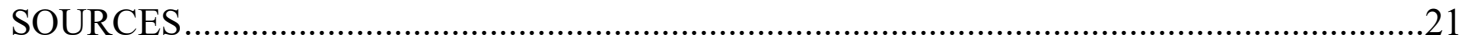

3.2 RECONSTRUCTION OF SIMULATED MEASUREMENT OF A FUEL ASSEMBLY .......24

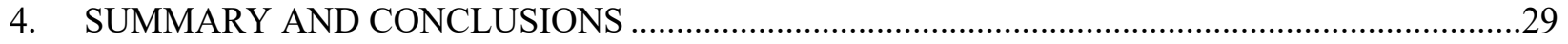

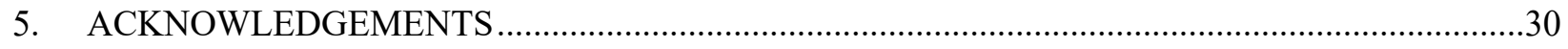

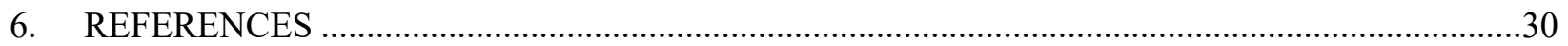





\section{LIST OF FIGURES}

Figure 1. The imager design is shown in plan view.....

Figure 2. Close-up schematic diagram of a single detector module where the detector edges can be seen.

Figure 3. The simulated response of each detector row to uniform illumination by a neutron source located at the center of the FOV when the collimator is not present.

Figure 4. The relative efficiency of an ideal detector module with minimal gap to the neighboring detector modules.

Figure 5. The (black) simulated and (red) empirical fit PSF for neutrons impinging on detector 158 from slit 49 on (left) a linear y-scale and (right) a logarithmic y-scale.

Figure 6. The results of (black) fits to the simulated PSFs and (red) the best linear trend for the (left) offset, (center) width, and (right) amplitude of the Gaussian fit.

Figure 7. The (black) simulated and (red) smoothed values of the collimator thickness as a function of detector number for a source positioned in the center of the FOV.

Figure 8. Schematic diagram illustrating path length through the collimator to the detector to calculate collimator penetration.

Figure 9. Schematic diagram showing the geometry used to calculate the contribution of slit scattering.

Figure 10. Schematic diagram of a single slit of outer opening $\boldsymbol{w}$ and length $\boldsymbol{t}$ a distance $\boldsymbol{d}$ from the line along which sources were positioned to calculate neutron response.

Figure 11. Normalized neutron response as a function of the distance of the source past the slit edge.

Figure 12. Normalized neutron response as a function of the scaled distance of the source past the slit edge.

Figure 13. Positions of (red line) slit 49 and (red points) the 35 different source positions for (left) a collimator with a single slit and (right) a collimator with 96 slits.

Figure 14. Comparison of (above) MCNP simulation and (below) analytical calculation of the imager response as a function of the source distance from the opening of slit 49 for (left) a collimator with only slit 49 and (right) the 96-slit collimator.

Figure 15. Simulated (black) and calculated (red) imager response for points 5, 10, 15, 20, 25, and $30 \mathrm{C}$ from the slit opening of a single-slit collimator where the slit is positioned in the same place as slit 49 .

Figure 16. Simulated (black) and calculated (red) imager response for points 5, 10, 15, 20, 25, and $30 \mathrm{~cm}$ from the slit opening of slit 49 .

Figure 17. Schematic diagram of the collimator with example response shown for neutron detections in detector number 158 .

Figure 18. Illustration of forward projection to calculate a sinogram from an image.

Figure 19. The positions of five line sources for the simulated tomographic imaging measurement are shown in red superimposed on a background of the layout of fuel pins in a $17 \times 17$ fuel assembly.

Figure 20. The (left) simulated and (right) reconstructed sinogram.

Figure 21. Comparison of MCNP simulation and MLEM reconstruction for (left) projection 8 and (right) projection 70 .

Figure 22. The image of five line sources reconstructed using the MLEM algorithm.

Figure 23. Comparison of the ability to resolve the closest sources with iteration number.

Figure 24. Resolution for line sources as a function of iteration for (black) neighboring sources and (red) next-to-neighboring sources.

Figure 25. Schematic diagram of the source geometry for the simulated $17 \times 17$ fuel assembly...............24

Figure 26. The (left) simulated and (right) reconstructed sinograms for the $17 \times 17$ fuel assembly. 
Figure 27. Comparison of MCNP-simulated and reconstructed sinograms for projection number 56

Figure 28. The reconstructed image of full fuel assembly.

Figure 29. The reconstructed image of the idealized sinogram (calculated using the imager response)

Figure 30. The position and sinogram for three example fuel pin positions.

Figure 31. Reconstructed neutron source values for fuel pins (left) shown as a $17 \times 17$ array and (right) as an idealized image with the fuel pins shaded by the appropriate value.

Figure 32. The (left) simulated and (right) reconstructed sinogram

Figure 33. Comparison of MCNP-simulated and pin-constrained reconstructed sinograms for projection number 56.

\section{LIST OF TABLES}

Table 1. Estimating functions for the amplitude, offset, and width of the Gaussian functions that model the PSF as a function of angle of incidence of the slit on the detectors (in degrees) 


\section{ACRONYMS}

$\begin{array}{ll}\text { FOV } & \text { field of view } \\ \text { FWHM } & \text { full width at half maximum } \\ \text { HDPE } & \text { high-density polyethylene } \\ \text { MLEM } & \text { maximum likelihood expectation maximization } \\ \text { ORNL } & \text { Oak Ridge National Laboratory } \\ \text { PSF } & \text { point spread function } \\ \text { PTI } & \text { Proportional Technologies Inc. }\end{array}$





\begin{abstract}
For the past three years, Oak Ridge National Laboratory (ORNL) has been developing a passive fastneutron emission tomography capability. The goal of this development is the ability to quantify the neutron source strength of individual fuel pins (rods) in spent nuclear fuel assemblies. Such a system could be used to measure the burnup of each fuel pin in a spent fuel assembly to take burnup credit when loading dry storage casks or to count individual fuel pins in spent fuel assemblies for safeguards purposes. At present, a laboratory prototype imager is under construction. The purpose of this prototype is to demonstrate imaging capability sufficient to resolve individual fuel pins in spent fuel assemblies. This report documents the development of the iterative reconstruction code used to perform tomographic image reconstruction, the imager response calculation used by the reconstruction code, and the results of reconstructions of simulated tomographic imaging measurements for the prototype imager design.
\end{abstract}

\title{
1. INTRODUCTION
}

ORNL is developing a new capability for passive fast-neutron emission tomography. The goal of this development is the ability to quantify the neutron source strength of individual fuel pins (rods) in spent nuclear fuel assemblies, the unit of special nuclear material in nuclear reactors. This technology could be used to measure the burnup of each fuel pin in a spent fuel assembly to take burnup credit for loading dry storage casks. This technology could also be used like the passive gamma emission tomography capability presently being evaluated by the International Atomic Energy Agency to detect the removal or substitution of individual fuel pins in spent nuclear fuel assemblies for international safeguards applications, such as to verify the integrity of an assembly before transfer to difficult-to-access storage [1-2]. At present, a laboratory prototype imager is under construction. The purpose of the prototype imager is to demonstrate sufficient capability to resolve individual fuel pins in a spent nuclear fuel assembly. This report documents the development of the iterative reconstruction code used to perform tomographic reconstruction, the imager response calculation used by the reconstruction code, and the results of reconstructions of simulated tomographic imaging measurements for the prototype imager design. This report fulfills the deliverable "Technical report on the image reconstruction technique and simulated performance of the imager for a number of fuel assemblies" for the project "Detection of Fuel Pin Diversion via Fast Neutron Emission Tomography" supported by the Office of Defense Nuclear Nonproliferation Research and Development.

Most readers are familiar with the notion of computed tomography from medical physics, where x-ray computed tomography has been commonplace for decades, and emission tomography modalities such as positron emission tomography and single photon emission computed tomography also see wide diagnostic use. In contrast, emission tomography using neutrons is a novel concept. Therefore, it is valuable to review aspects of computed tomography that relate to the working principles of the fastneutron imager.

In each form of computed tomography, projection data from many angles are used to reconstruct crosssectional images of an inspected object for diagnostic purposes. The crucial elements of this process are that measured data divide the object into "lines of response," and multiple views through the object are mathematically combined to estimate an image. Here, a line of response refers to a path through the inspected object along which an observable (such as neutron emission activity) can be integrated. For passive neutron imaging, a collimator slit defines the line of response through the fuel assembly, and neutron counts in a detector at the terminus of a collimator slit correspond primarily to an integral of neutron activity along the path defined by the slit. It is possible to achieve fast-neutron tomography with the desired resolution provided each line of response sufficiently isolates a path through the object, the object is sampled with a sufficient number of lines of response, and there are a sufficient number of views 
through the object to invert measured data to form an image. For tomographic images of a spent nuclear fuel assembly, the neutron emission from each fuel pin can be extracted from the reconstructed image.

The use of fast neutrons to perform tomography is desirable because

- neutrons readily penetrate the high-atomic-number fuel in the assemblies, making the contribution of neutrons from fuel pins from the interior of the assembly readily measurable;

- neutrons, originating from actinides, are more linked to the fissile material content of the fuel than gamma rays;

- neutron measurements provide a potential pathway to nondestructively measuring plutonium effective for each fuel pin in a nuclear fuel assembly; and

- neutron measurements are more sensitive to assemblies containing fuel pins that were replaced after a single cycle in the reactor and subsequently irradiated in the core. This sensitivity is a consequence of the neutron signal originating primarily from the ingrowth of ${ }^{244} \mathrm{Cm}$, which occurs predominantly at the end of an exposure cycle.

In practice, neutron emission tomography was not developed earlier because of the scarcity of neutronemitting materials (they are only made as by-products of the nuclear fuel cycle) and the modest intensities of typical neutron sources compared to the corresponding gamma ray intensities. Moreover, the ability of fast neutrons to penetrate a good deal of shielding makes them difficult to collimate and measure with the required resolution. In previous work, ORNL demonstrated proof-of-concept emission-neutron computed tomography using fast fission neutrons with high-resolution scintillation detectors, but this approach is not suitable for the overwhelming gamma-ray emissions from spent fuel [3].

To address the combined challenges associated with effectively collimating neutrons, achieving sufficient resolution, maintaining sufficient efficiency for the modest neutron source strength from spent fuel, and maintaining sufficient insensitivity to the gamma-ray flux to allow meaningful neutron measurements, a new collimator concept has been developed termed a "parallel-slit ring collimator." This report opens with a description of the parallel-slit ring collimator. Then, the model that generates the neutron response of the collimator is described. Finally, the results of tomographic reconstructions of simulated data using the calculated response to neutrons are reported. A previous report details how the design of the present parallel-slit ring collimator was selected from similar designs with different parameters (such as slit width) to maximize the signal-to-noise ratio in the measured data [4].

\section{PARALLEL-SLIT RING COLLIMATOR}

Measuring fast neutrons is desirable because neutrons penetrate a good deal of shielding and readily escape from a fuel assembly, but their ability to penetrate shielding makes fast neutrons difficult to collimate and measure with fine spatial resolution. In addition, measuring the modest neutron source strength from spent fuel among the overwhelming gamma-ray emissions poses additional challenges. The challenges of using fast neutrons manifest themselves such that attributes of a detector or collimator that maximize one desirable characteristic (such as resolution) conflict with other desirable characteristics (such as efficiency). This work uses the functional equivalent to a parallel-slit collimator called a "parallel-slit ring collimator," which can be used with large detector pixels in close proximity to the fuel. In this design, the slits of a parallel-slit collimator are rearranged to distribute them around a ring that surrounds the target fuel assembly. This rearrangement allows for the necessary increased separation of collimator slits and the use of larger moderated neutron detectors. Moderated detectors offer the 
advantage of achieving their neutron efficiency in proportion to the volume of moderator, which is insensitive to gamma rays, while having comparatively little active volume that is sensitive to gamma rays.

The parallel-slit ring collimator design simultaneously addresses the following challenges:

- Gamma-ray dose management: Managing the gamma-ray dose rate requires a combination of gamma blind detectors and a collimator that effectively shields gamma rays. The requirement for gammablind neutron detectors points toward moderated detectors where most of the energy loss is in moderator rather than in active detector volume. Moderated detectors, in turn, imply large detectors. Gamma dose rate can be reduced with denser collimator materials, such as steel or lead, but these materials are poor neutron moderators.

- Resolution: Defining a line of response similar in size to the gap between fuel pins requires a slit width comparable to the gap dimension and a thick collimator from a good neutron moderator (like borated polyethylene). However, good neutron moderators do little to reduce gamma dose.

- Sufficient sampling: About 100 slits are necessary to sample the $35 \mathrm{~cm}$ field of view (FOV) of the imager with the required resolution.

- Efficiency: The modest neutron source strength of spent fuel requires efficient use of the available neutrons, such as by placing large detectors close to the fuel and using wide collimator slits. However, the modest neutron source strength also suggests that a sparse collimator design (implied by large detectors) combined with scanning would take too long but removing collimator material to add slits or make them wider reduces the effectiveness of the remaining slits.

- Compact overall size: For a number of detectors of specified size, a ring is the most compact geometry.

Use of the parallel-slit ring collimator concept makes it possible to construct an imager with sufficient resolution to identify individual fuel pins and keep the imager sufficiently compact, efficient, and radiation resistant to be practical. This collimator concept enables use of large detectors that are relatively close to the fuel with widely spaced collimator slits that simultaneously sample the entire volume.

The parallel-slit ring collimator design achieves the required spacing between slits (and detector elements) by rotating the position of each slit in a conventional parallel-slit collimator through a known angle to achieve equal detector spacing around the outside of the collimator annulus. In this way, the essential function of the collimator is maintained and correspondence to the parallel-slit collimator is maintained (i.e., isolating lines of response along particular chords across the central volume of the annulus). Each slit creates the same line of response as it would in its original parallel-slit geometry but for a different projection angle (rotation of the object). For tomographic measurements, all projection angles (spaced around $360^{\circ}$ ) are measured, so an equivalent set of views will be acquired for the rotated slits as for the original parallel slits. Also, for this collimator design, the fuel and detectors can remain stationary, and only the collimator need rotate. As a result, fuel-detector positioning does not have to be made very precisely provided there is no relative motion during the measurement. 


\subsection{IMAGER DESIGN}

The imager consists of an annular collimator surrounded by a ring of detectors. The annular collimator has a set of slits in it that define lines of response across the imager FOV. Spent nuclear fuel can be loaded in the central void of the annulus. The collimator modulates neutrons emitted by the fuel, and the modulated pattern of collimated neutrons are detected by the ring of neutron detectors. To perform imaging measurements, the collimator rotates, and the detectors and fuel remain stationary. Data for each chord across the FOV and each angle are recorded, enabling tomographic reconstruction. A schematic diagram of the imager design is shown in plan view in Figure 1. The outer diameter of the neutron imager is $148 \mathrm{~cm}$. In the dimension extending out of the page, the neutron imager extends $100 \mathrm{~cm}$.

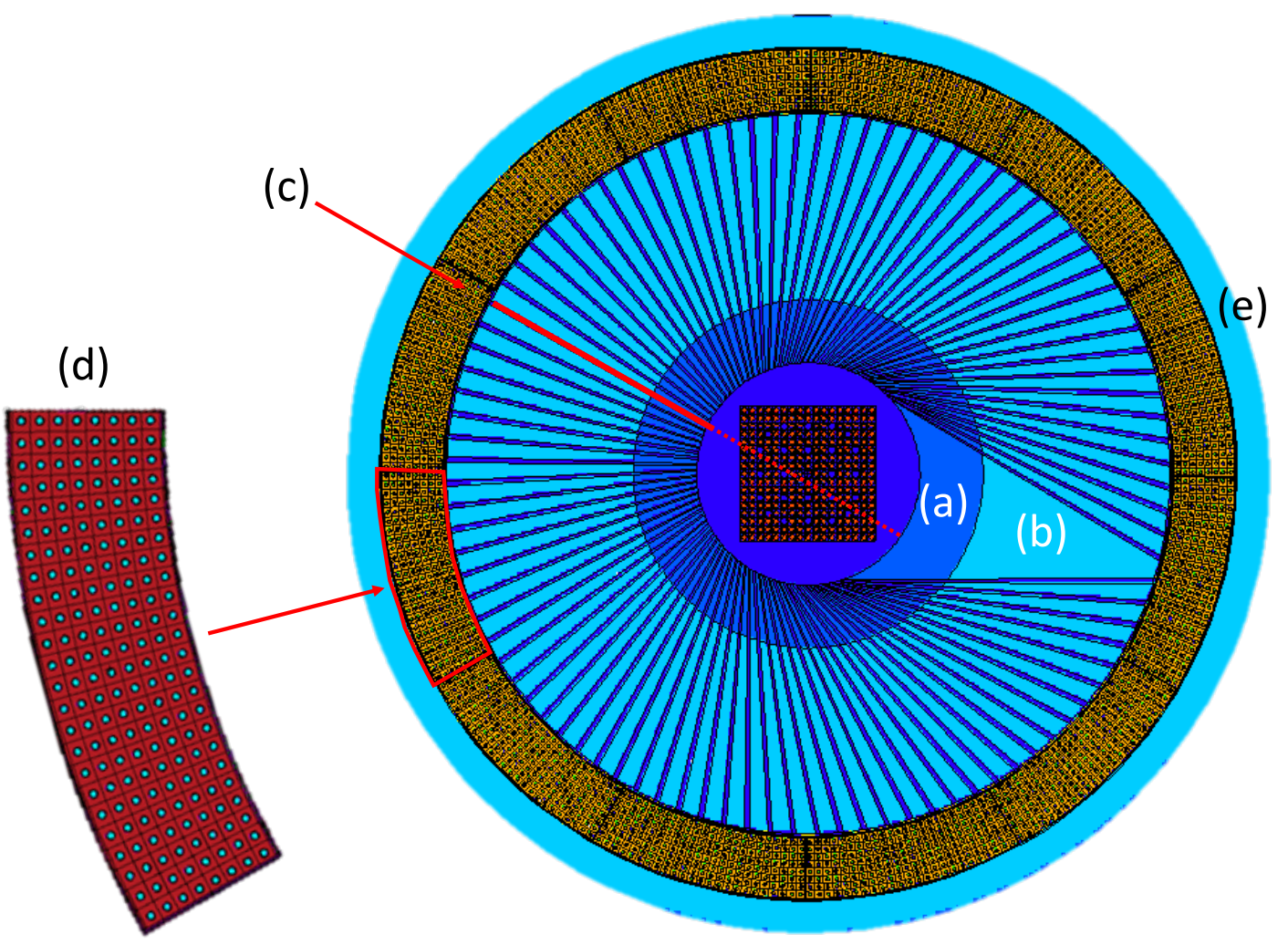

Figure 1. Plan view of the imager design. The collimator annulus is composed of an (a) inner stainless steel and (b) outer borated polyethylene ring and has (c) 96 slits in it that define lines of response across the FOV. Neutron counts are recorded in (d) 12 detector modules that each have 24 rows of 8 boron straws. The detector ring is shielded by (e) a $5 \mathrm{~cm}$ thick ring of borated polyethylene.

The inner radius of the collimator annulus is $17.67 \mathrm{~cm}$. This dimension is intended to accommodate most common light water reactor fuel assemblies. In particular, the inner diameter was chosen such that its inscribed square is $1 \mathrm{~cm}$ greater in side length than an assembly with a width of $24 \mathrm{~cm}$. The outer diameter of the imager was chosen so that it can be readily handled by a facility, so its size was constrained to fit in a cask or cask-like container that nuclear operators typically handle. The outer diameter was therefore chosen to fit within the internal cavity of the $148 \mathrm{~cm}$ internal cavity diameter of the GNS Castor series of transport casks, a cask type commonly used for international fuel shipments.

The collimator annulus is made up of an inner stainless-steel portion and an outer borated polyethylene portion. The stainless-steel portion of the collimator annulus is $10 \mathrm{~cm}$ thick. This portion provides 
structural integrity and shielding of the neutron detectors from the intense gamma-ray field emitted by the fuel. The borated polyethylene portion of the collimator annulus is $29.5 \mathrm{~cm}$ thick. This portion modulates fast neutrons for imaging.

The collimator has 96 slits defining lines of response that traverse the FOV. The number of slits in the collimator was chosen such that there are sufficient lines of response to sample the gaps between fuel pins. Common $17 \times 17$ fuel assemblies have inter-pin gaps of $0.328 \mathrm{~cm}$. Sampling $96 \%$ of the inner diameter of the collimator at a spacing equaling the inter-pin gap requires approximately 100 slits; 96 is the closest multiple of 16 (a common unit of modular electronics) to 100 . Tapered slits ( $3 \mathrm{~mm}$ wide at the collimator inner radius and $8 \mathrm{~mm}$ wide at the collimator outer radius) control gamma dose while maintaining neutron efficiency.

The ring of detectors records neutrons that traverse the slits. The detector ring consists of 12 modules, each with 24 rows of 8 boron straw detectors embedded in high-density polyethylene (HDPE) moderator. The annular design of the detector produces the most compact size for the number of detectors. Tolerance to gamma dose dictated that each row of 8 straws is read separately to yield 24 channels per module and a total of 288 channels for the imager. Note, the number of detector channels oversamples the number of slits by a factor of three.

Each straw in the detector is an approximately $4 \mathrm{~mm}$ diameter gas-filled aluminum tube that contains a ${ }^{10} \mathrm{~B}_{4} \mathrm{C}$-coated copper straw at the outer diameter and an anode wire in the center. Neutrons are detected via the neutron capture reaction ${ }^{10} \mathrm{~B}+n \rightarrow{ }^{7} \mathrm{Li}+\alpha$ reaction when either the $0.84 \mathrm{MeV}{ }^{7} \mathrm{Li}$ or $1.47 \mathrm{MeV}$ $\alpha$ escapes the ${ }^{10} \mathrm{~B}_{4} \mathrm{C}$ coating and creates sufficient ionization in the fill gas of the straw to exceed the detector threshold. The straws are resilient to high gamma-ray fields because (1) most of the detector consists of inert moderator, (2) the small gas volume per straw and low gas pressure minimize the amount of material where gamma rays (and scattered electrons) can interact, and (3) the small radius of the detector means that the signals are inherently fast (tens of nanoseconds rather than microseconds) to minimize the amount of gamma-ray pileup. The ${ }^{10} \mathrm{~B}$ straws are manufactured by Proportional Technologies Inc.. Although the detectors detect neutrons at thermal energies, by incorporating boron in the collimator and placing a cadmium thermal-neutron filter between the collimator and detector, the imager still responds primarily to fast neutrons. As instrumented, the boron straw detectors can tolerate gamma fields up to approximately $500 \mathrm{R} / \mathrm{h}$.

Outside the detector ring, there is a ring of borated polyethylene shielding to reduce the background rate of neutrons in the detectors from potential surrounding neutron sources.

\subsection{NEUTRON RESPONSE MODEL}

Iterative image reconstruction corresponds to estimating the image $\boldsymbol{x}$ that generates measured data $\boldsymbol{y}$ given imager response $\boldsymbol{A}$. The process of converging to a solution entails many calculations of trial measurements, $\boldsymbol{y}_{\text {trial }}$, given a trial image $\boldsymbol{x}_{\text {trial }}$ via $\boldsymbol{y}_{\text {trial }}=\boldsymbol{A} \boldsymbol{x}_{\text {trial }}$. For the present imager, the response $\boldsymbol{A}$ encodes the expected counts per source neutron recorded in each detector for each position in the FOV and any rotation angle of the collimator. Calculating this on a millimeter grid for neutron imaging measurements with 96 discrete rotation angles (one corresponding to each slit) requires calculation of $2.7 \times 10^{9}$ values.

Potential approaches to generating the response include

- generating the response at each point using an MCNP radiation-transport simulation; 
- generating the response via MCNP radiation-transport simulation on a sparser grid, then interpolating; or

- using relatively few MCNP radiation transport simulations to understand the response in terms of analytical functions suggested by physical principles with empirically fit parameters that describe the response sufficiently well.

In the present work, the first approach would require about $10^{7}$ simulations, each with at least $10^{8}$ source histories. Although possible, this approach was deemed too computationally expensive. The second approach would work well for the slowly varying components of the response but not for the rapidly changing part encoding the lines of response of the slits. As a result, an analytical description for the neutron response was developed. The description is inspired by the expected physical processes, then empirically fit to extract the best agreement with data from a limited number of MCNP simulations. Note, the response needs to capture all spatial variations that would otherwise be attributed to differences in neutron intensity. For the reconstruction of full $17 \times 17$ arrays, a single fuel pin corresponds to less than $0.4 \%$ of the total intensity, so small inaccuracies in the response can be important.

For development of the neutron response, a number of components of the response were identified, and radiation transport simulations were performed to quantify them. These components included

- efficiency variation across individual straw rows of the neutron detectors;

- inter-detector scattering in which neutrons traverse one slit, then scatter into different detectors where they are subsequently detected (also known as the "point spread function" or PSF);

- a "collimator penetration" component, where neutrons traverse the collimator similarly to a solid (but lower density) collimator;

- a "slit scattering" component, where neutrons are originally incident on the vicinity of the collimator near the slit opening, then scatter down the slit;

- a "direct transmission" component consisting of direct illumination of the detector by the source, unimpeded by the collimator slit; and

- an "edge transmission" component where the slit wall either partially or fully blocks the direct path to the detector but average paths from the source to the detector through the collimator are substantially smaller than the full collimator thickness.

To develop the response, radiation transport simulations were performed using MCNP6. For all of the simulations, ${ }^{244} \mathrm{Cm}$ fission was used to define the neutron source energy spectrum. Neutron counts in the boron straws were recorded via an F4 tally with a tally multiplier to convert to reaction rate in the boron carbide and then multiplied by the volume to convert to counts per source neutron. Typical simulations consisted of $10^{8}$ source histories.

\subsubsection{Detector efficiency variations}

The efficiency of each row of eight straws is not expected to be identical across every detector module. Experimentally, the response of individual straw rows may differ because of small variations in the boron carbide layers in individual straws or small variation in the gain of notionally identical electronics. In addition to these differences, the efficiency of the straw rows near the edges of the detector modules will be lower because of the existence of a gap in the HDPE moderator between straw rows on either side of 
the boundary between two modules. In Figure 2, a schematic diagram of the imager that is zoomed to show a single detector module and its neighbors illustrates that the amount of HDPE in the vicinity of the edge straw rows is reduced. As a result, the efficiency of the rows of straws in the vicinity to the edge is reduced.

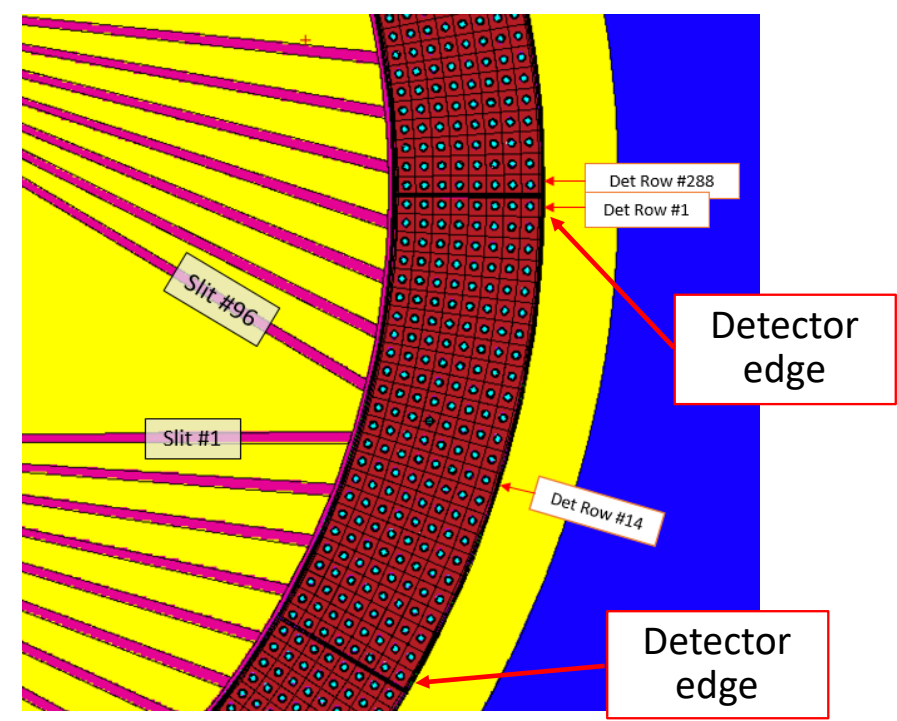

Figure 2. Close-up schematic diagram of a single detector module, showing the detector edges.

Radiation transport simulations using MCNP6 were performed for a line source of ${ }^{244} \mathrm{Cm}$ located at the center of the imager FOV with the collimator not present. The resulting variation in detected counts per source neutron as a function of detector number is displayed in Figure 3. Note the drop in efficiency near the edges of the detectors.

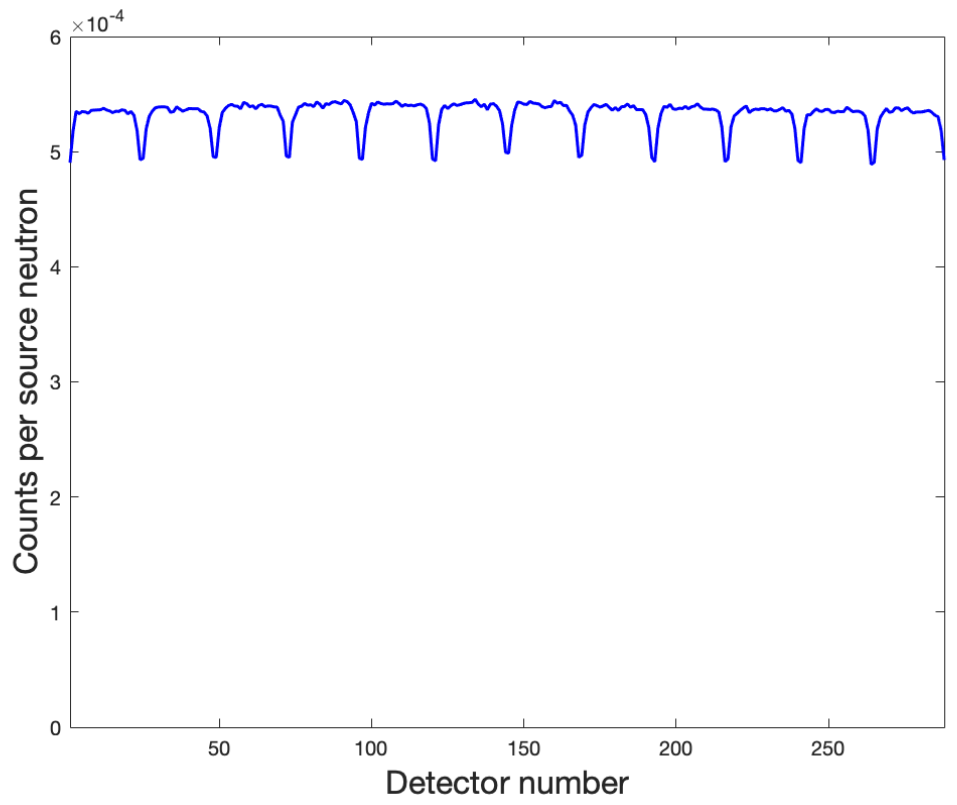

Figure 3. Simulated response of each detector row to uniform illumination by a neutron source located at the center of the FOV when the collimator is not present. 
In these simulations, each detector module is identical, and the gaps between the detector module and its neighbor are minimal. As a result, a statistically more precise efficiency map is possible by averaging the response of each detector module. This average detector response is shown in relative terms in Figure 4. The loss of efficiency in the straw rows at the edge of a detector module is about $8 \%$ compared to straw rows in the center of the detector. For comparison, the deficit in neutron counts when looking down a row of 17 fuel pins with one missing might be expected to be $5.9 \%(1 / 17)$. In the laboratory prototype imager, the efficiency map of the detector will be directly measured using a neutron source.

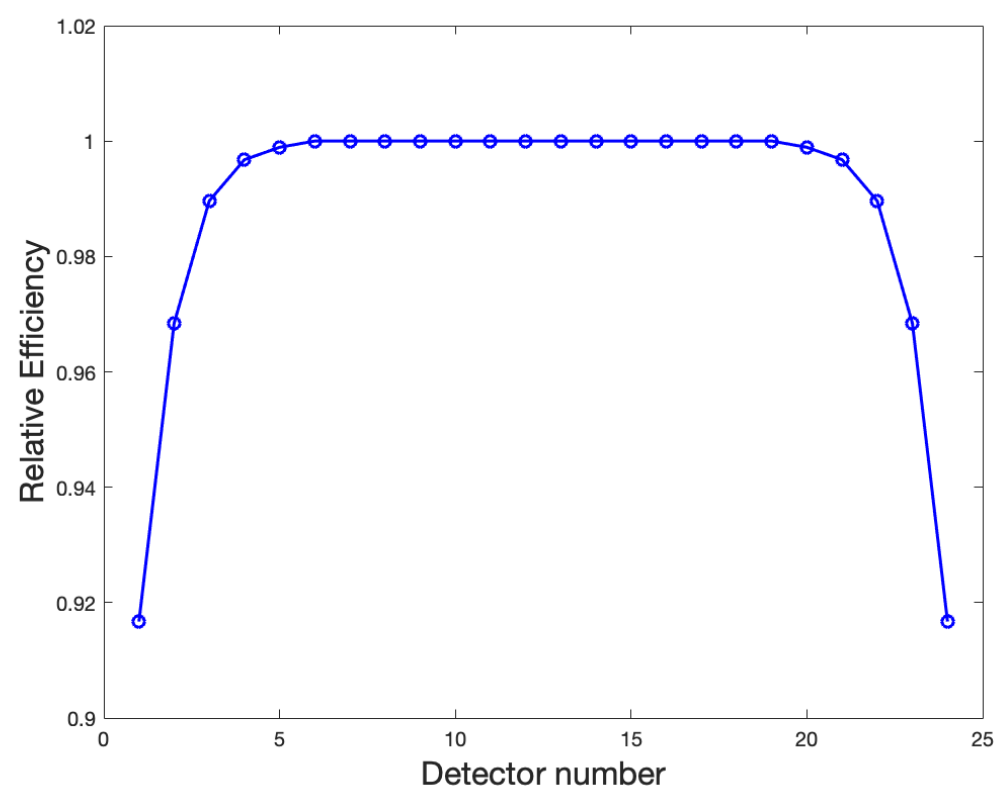

Figure 4. Relative efficiency of an ideal detector module with minimal gap between the neighboring detector modules.

\subsubsection{Point spread function (PSF)}

One of the biggest factors limiting the resolution of the imager is inter-detector scattering. Inter-detector scattering occurs when neutrons traverse one slit and then scatter from the intended detector to another before being detected. In a true parallel-slit collimator, inter-detector scattering has the same effect as lower spatial resolution because in either case, a fraction of detected neutrons associated with a particular collimated path are misattributed to a nearby path. Although the physical process producing the reduced spatial resolution is referred to as inter-detector scattering, the characteristic spreading of the detector response to a beam of neutrons impinging on its surface is called the "point spread function" or PSF. To characterize the PSF in any part of the detector with the collimator in any orientation, simulations were performed using a beam of neutrons that has the energy spectrum of ${ }^{244} \mathrm{Cm}$ spontaneous fission directed down each slit of the collimator, and the neutron capture reaction rate in each straw was recorded. The results of these simulations were analyzed to extract an empirical description. An example simulated PSF is shown in Error! Reference source not found. by the black points for slit 49 pointing to detector number 158. 

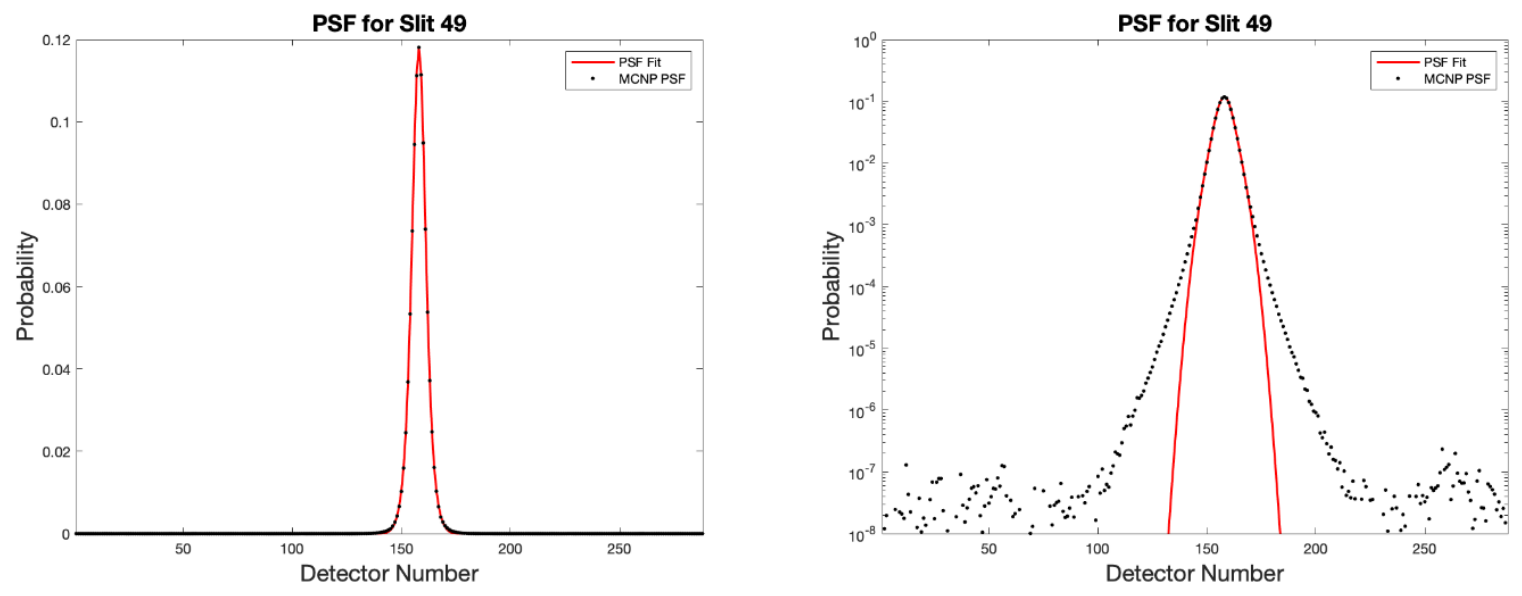

Figure 5. (Black) Simulated and (red) empirical fit PSF for neutrons impinging on detector 158 from slit 49 on (left) a linear y-scale and (right) a logarithmic y-scale.

The resulting characterization by a double Gaussian fit is shown in red, which matches the simulated data to $1 \%$ of the peak height. The characterized PSF is represented by the following equation:

$$
P S F=A(\theta)\left(\exp \left(-\frac{\left(x-x_{0}(\theta)\right)^{2}}{2 \sigma(\theta)^{2}}\right)+\frac{1}{1.75} \exp \left(-\frac{\left(x-x_{0}(\theta)\right)^{2}}{2(1.75 \sigma(\theta))^{2}}\right)\right)
$$

where $A, x_{0}$, and $\sigma$ represent the amplitude, offset, and width of the distribution, and $x$ represents the distance (in detectors) between the detector at the outer diameter of the slit and the detector where the response peaks. Here, $A, x_{0}$, and $\sigma$ have been written as a function of the angle $\theta$ between the slit and the unit normal of the detector surface to emphasize that their values depend on that angle. Importantly, the distance $x$ between neighboring detectors at the edge of detector modules must be reduced from unity to 0.8716 to make the parameters a relatively smooth function of the angle $\theta$. This shorter distance reflects the reduced amount of HDPE moderator at the edges of the detector, so the distance $x$ more accurately reflects an effective distance in HDPE moderator. When $x$ has been modified in this way, the (right) amplitude, (center) width, and (left) offset are shown as a function of the slit angle by the black points in Figure 6 . The corresponding best-fit lines are shown in red and the fit values are tabulated in Table 1.

Table 1. Estimating functions for the amplitude, offset, and width of the Gaussian functions that model the PSF as a function of angle of incidence of the slit on the detectors (in degrees).

\begin{tabular}{|c|l|}
\hline Parameter & \multicolumn{1}{c|}{ Estimated function } \\
\hline$A(\theta)$ & $7.3569 \times 10^{-2}+2.7192 \times 10^{-4} \cdot \theta$ \\
\hline$\sigma(\theta)$ & $2.6312+1.221 \times 10^{-2} \cdot \theta$ \\
\hline$x_{0}(\theta)$ & $1.5664 \times 10^{-4}+5.9349 \times 10^{-2} \cdot \theta$ \\
\hline
\end{tabular}



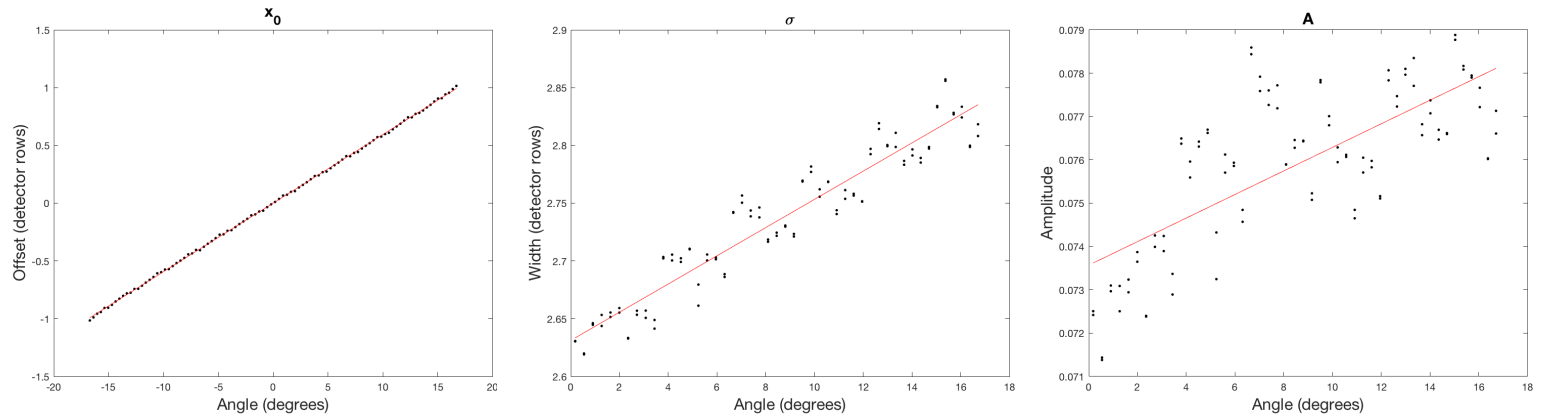

Figure 6. Results of (black) fits to the simulated PSFs and (red) the best linear trend for the (left) offset, (center) width, and (right) amplitude of the Gaussian fit.

The approximate PSF for each slit can be calculated for any orientation of the collimator simply by knowing the angle of each slit and the detector it is pointing toward.

\subsubsection{Collimator penetration}

The collimator penetration portion of the response quantifies the contribution of neutron transmission along nonpreferential paths through the collimator. This nonpreferential transmission can be thought of as similar to transmission through a solid but lower-density collimator. It is most straightforward to express the amount of transmission through the collimator in terms of an effective thickness, which is given in terms of the number of mean free paths. The effective thickness of the collimator can be inferred from the negative logarithm of the ratio of counts in the collimator penetration portion of the response to the corresponding value with no collimator present. This process isolates the effects of the collimator and eliminates the contributions of the solid angle and backscatter from the borated polyethylene shield.

Because the collimator slits are unequally distributed around the inner diameter of the collimator, there is not a single value for the effective collimator thickness. Rather, there is a local value of the collimator depending on the density of slits along a path between the source and each detector. In principle, this value depends on both the source and the detector positions with respect to the orientation of the collimator. At present, the effective thickness is calculated for the source positioned in the center of the FOV. The effective thickness is shown in Figure 7 for (black) simulated data and (red) a smoothed local fit to the data. 


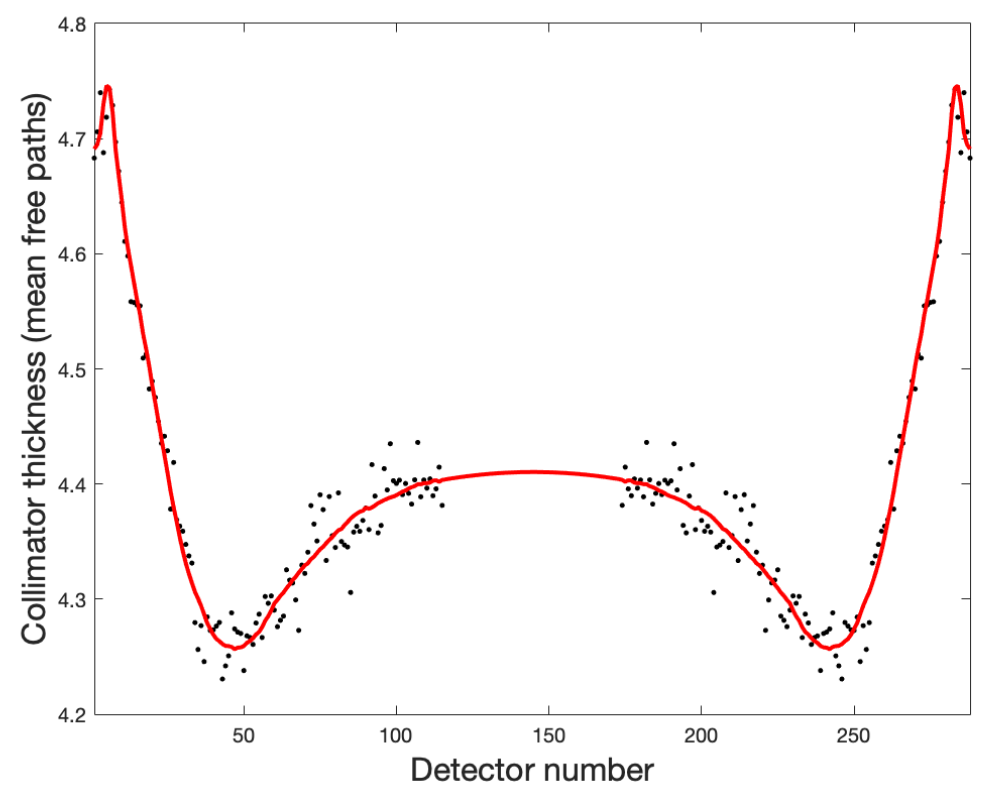

Figure 7. (Black) Simulated and (red) smoothed values of the collimator thickness as a function of detector number for a source positioned in the center of the FOV.

From this value of effective thickness, or $t_{e f f}$, the amount of collimator penetration can be estimated for each detector and position of the source. Figure 8 illustrates that for an arbitrary position of the source, the path through the borated polyethylene portion of the collimator has length $t+\Delta t$ instead of the borated polyethylene collimator thickness $t$. This collimator path will have an effective thickness of

$$
\Delta t_{e f f}=t_{e f f} \cdot \frac{\Delta t}{t}
$$

where

$$
\frac{\Delta t}{t}=\frac{R_{\text {out }} \cos \theta-\sqrt{r_{\text {in }}^{2}-R_{\text {out }}^{2} \sin ^{2} \theta}}{R_{\text {out }}-r_{\text {in }}}-1 .
$$

Here, $R_{\text {out }}$ is the outer radius of the borated polyethylene, $r_{i n}$ is the radius to the inner radius of the borated polyethylene, and $\theta$ is the angle between rays pointing from the detector to the source and from the detector to the FOV center. 


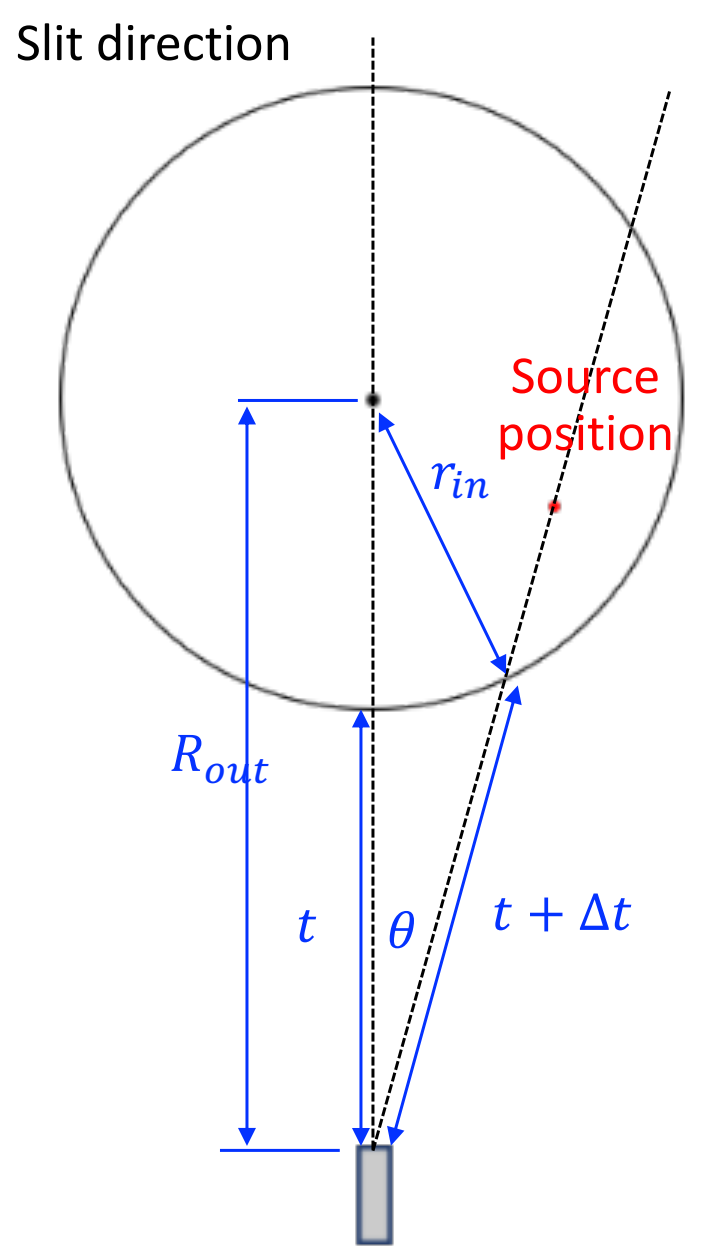

Figure 8. Schematic diagram illustrating path length through the collimator to the detector to calculate collimator penetration.

Given that $A_{c p} \exp \left(-t_{e f f}\right)$ yields the value of collimator penetration with the source in the middle of the FOV, the factor $\left(\frac{R_{\text {out }}}{R}\right) \exp \left(-\Delta t_{\text {eff }}\right)$ corrects for different source-detector distances and paths through the collimator. Then, the collimator penetration $P$ for any point in the FOV can be estimated by

$$
P=A_{c p}\left(\frac{R_{\text {out }}}{R}\right) \exp \left(-\Delta t_{\text {eff }}\right) \exp \left(-t_{\text {eff }}\right)
$$

\subsubsection{Slit scattering}

Slit scattering refers to the process by which some neutrons originally incident on the region of the collimator near the slit opening subsequently scatter one or more times in the collimator and are redirected along an unimpeded path down the slit to the detector. The probability is inversely proportional to the distance from the source to the slit opening $r$ and the length of the slit $s$. 


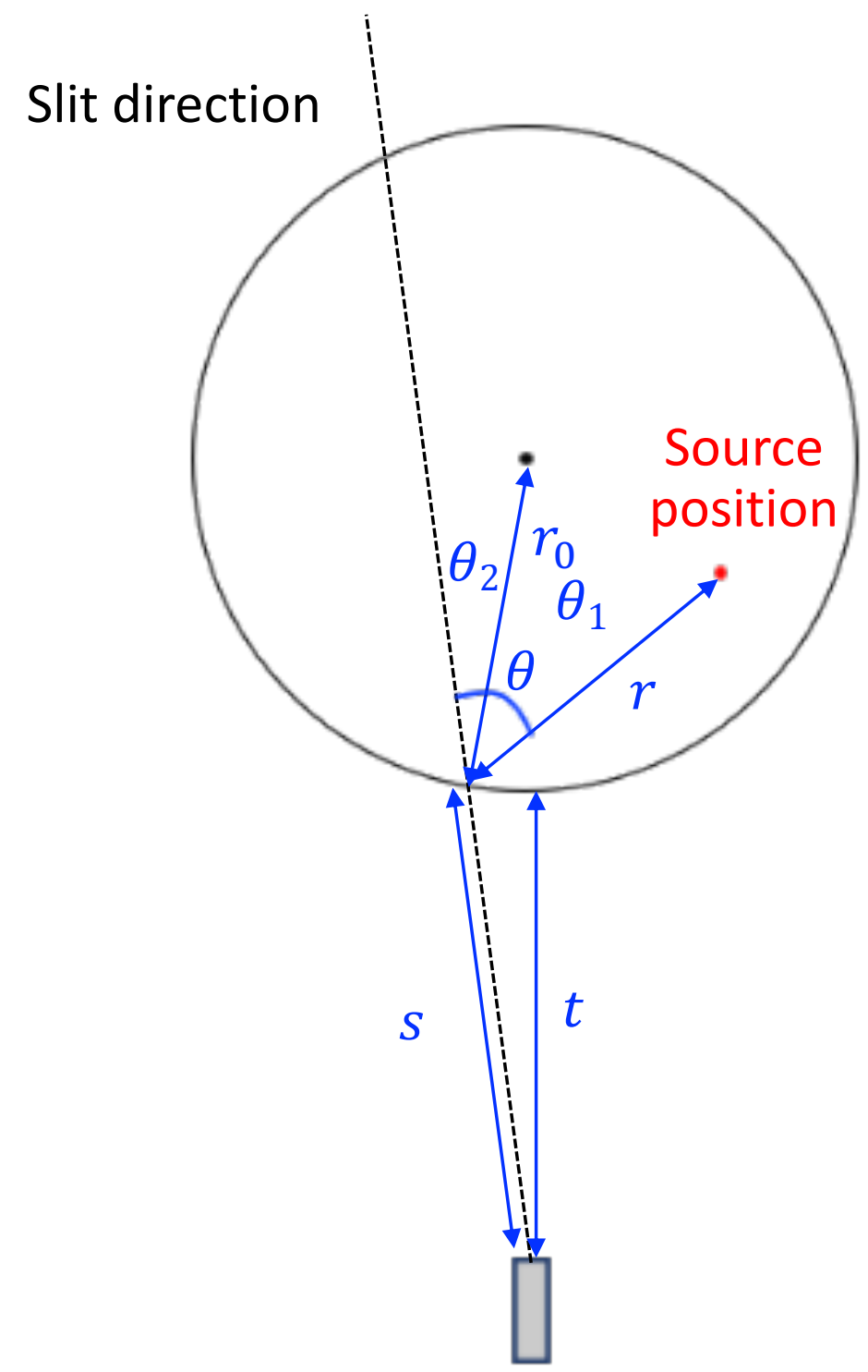

Figure 9. Schematic diagram showing the geometry used to calculate the contribution of slit scattering.

The slit scattering probability is also approximately proportional to the cosine of the angle between the incident neutron path and the slit direction $(\cos \theta)$. As modeled, the slit scattering, $S$, can be written as

$$
S=A_{s} \frac{t}{S} \cdot \frac{r_{0}}{r+2} \cdot\left\{\begin{array}{c}
\cos \theta, \cos \theta>\cos \theta_{1} \cos \theta_{2} \\
\cos \theta_{1} \cos \theta_{2}, \text { otherwise }
\end{array} .\right.
$$

Note, the 2 in the denominator of $r_{0} /(r+2)$ is in centimeters and is present because the neutron only needs to be incident near the slit opening. Here, $r_{0}$ refers to the inner diameter of the collimator, not the inner diameter of the borated polyethylene (referred to earlier as $r_{i n}$ ). Also, it is possible to have angles between the slit vector and the slit-to-source vector that exceed $90^{\circ}$. In these cases, it is desirable that the slit scattering still have a positive contribution, not a negative one. Therefore, the cosine of the angle between the slit vector and the slit-to-source vector is replaced by the product of the cosine of the angle between the slit vector and the slit-to-FOV-center vector $\left(\cos \theta_{2}\right)$ and the cosine of the slit-to-FOV-center vector and the slit-to-source vector $\left(\cos \theta_{1}\right)$. 


\subsubsection{Direct and edge transmission}

The direct and edge responses of the collimator quantify neutron transmission along preferential paths through the collimator. The "direct transmission" component consists of direct illumination of the detector by the source, unimpeded by the collimator slit. The "edge transmission" component consists of the response where the slit wall either partially or fully blocks the direct path to the detector, but the length of contributing paths from the source to the detector through the collimator are substantially smaller than the full collimator thickness.

To investigate the direct and edge components of the response, neutron transport simulations were performed for a collimator with a single slit in it and a ${ }^{244} \mathrm{Cm}$ line source positioned at several locations along a line perpendicular to the centerline of the single slit at three different distances from the slit opening: 5.04, 10.08, and $20.16 \mathrm{~cm}$. For the simulations associated with each distance from the slit opening, 89 different source positions were simulated spanning the distance from the slit centerline of $-3.5 \mathrm{~cm}$ to $+3.5 \mathrm{~cm}$ in $0.1 \mathrm{~cm}$ steps and further extending to $-8 \mathrm{~cm}$ and $+8 \mathrm{~cm}$ from the slit centerline in $0.5 \mathrm{~cm}$ steps. For each set of simulations, the component of the response corresponding to collimator penetration was subtracted and the subtracted values normalized to the intensity of the simulation along the slit centerline (position 0).

A representative line at distance $d$ from the opening of the slit of opening $w$ and length $t$ is shown schematically in Figure 10.

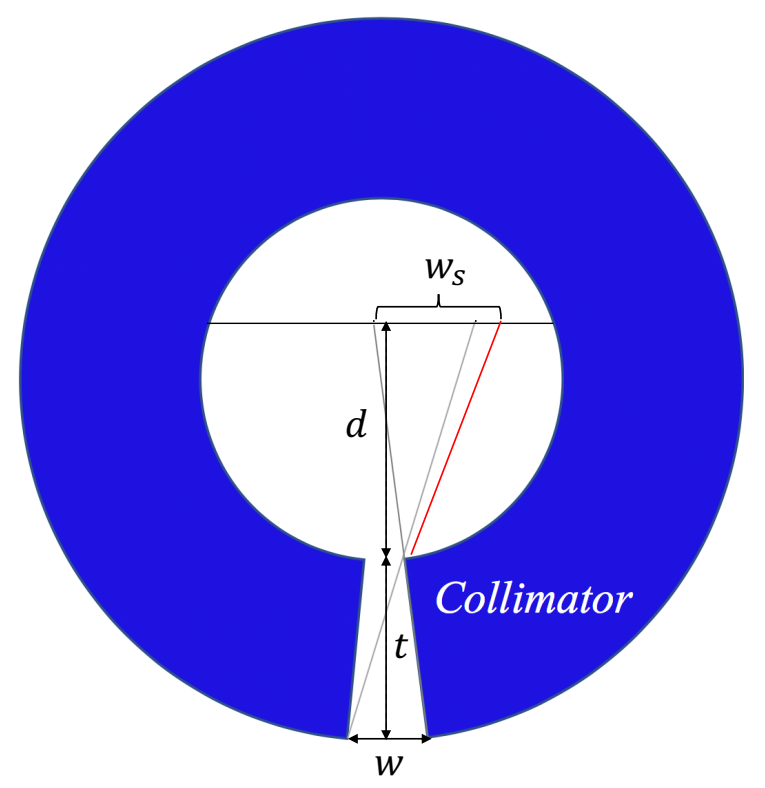

Figure 10. Diagram of a single slit of outer opening $w$ and length $t$ a distance $d$ from the line along which sources were positioned to calculate neutron response.

The drop off in the response as the source moves past the slit edge is shown in Figure 11 for each distance. Note, the shape of each response is similar but scaled in proportion to the distance from the slit. 


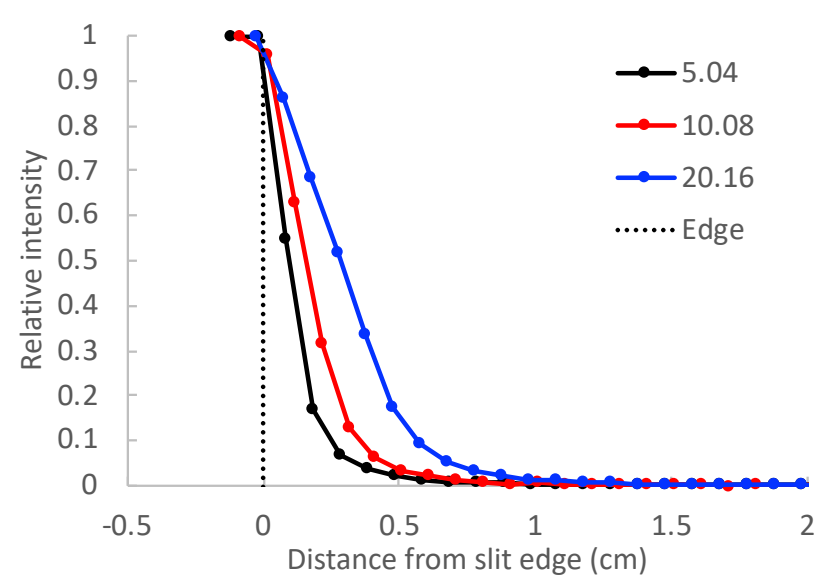

Figure 11. Normalized neutron response as a function of the distance of the source past the slit edge.

The response to the source moving past the slit edge is nearly identical if the distance past the slit edge $x$ is scaled by the value $a_{0} \frac{w}{t} d+a_{1} \frac{w}{t}$, where $a_{0}=1.1896$ and $a_{1}=3.7057$. This line is approximately indicated in red in Figure 10 and corresponds to the location where the neutron response drops to near zero. When the normalized neutron response is plotted as a function of the distance from the edge scaled by this quantity, the responses at the three distances lie along the same line as shown in Figure 12. This line drops from a maximum value when aligned with the slit edge to a value near 0 at a scaled distance of unity.

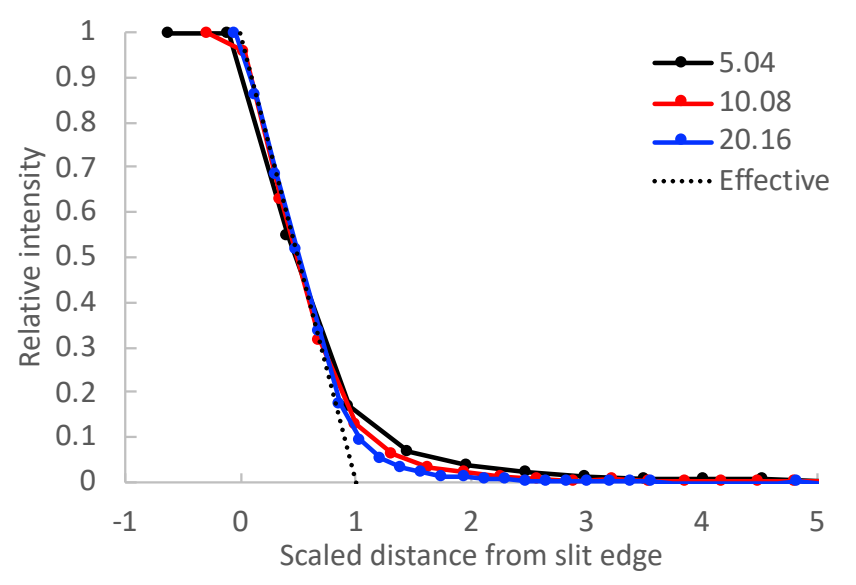

Figure 12. Normalized neutron response as a function of the scaled distance of the source past the slit edge. The scale factor is described in the text.

The response in the slit edge region can therefore be modeled as the response neglecting the slit edge multiplied by a factor $f$ between 0 and 1 that equals

$$
f=1-\frac{x}{a_{0} \frac{w}{t} d+a_{1} \frac{w}{t}}
$$

Equation (4) is valid if the distance $x$ is on the collimator side of the slit and its value is less than or equal to $a_{0} \frac{w}{t} d+a_{1} \frac{w}{t}$. 
The response where the source has unimpeded view of the entire back opening $w$ should, in principle, scale like the inverse of the distance from the source to the detector. However, this is slightly complicated by the side of the slit at the collimator inner diameter having a different solid angle than the side of the slit at the collimator outer diameter. In practice, we take this into account by allowing an exponent for the inverse distance from the detector that can be greater than unity. Then, the direct (and edge) components can be written as

$$
D=A_{d}\left(\frac{R_{\text {out }}}{d+t}\right)^{\alpha} f_{1} f_{2}
$$

where $R_{\text {out }}$ is the outer diameter of the collimator, $t$ is the thickness of the collimator, and $d$ is the distance (along the slit) from the slit opening to the source. The factors $f_{1}$ and $f_{2}$ are calculated for each slit edge using Equation (4). The exponent $\alpha$ is tuned to match data, and the value $\alpha=1.43$ is optimum for matching the radiation transport simulations of a collimator with single slit and a collimator with 96 slits in it.

\subsubsection{Comparison of the neutron response model to simulation}

The total neutron response $T$ was calculated as the sum of the direct, collimator penetration, and slit scattering components. The direct and slit scattering components are operated on by the PSF. All components are operated on by the relative detector efficiency, $\epsilon_{\text {rel }}$. This can be written

$$
T=\epsilon_{r e l} \circ(P S F \cdot D+P S F \cdot S+P) .
$$

In this equation, the operator "o" refers to elementwise multiplication while "." refers to matrix multiplication. The expression $D$ should be interpreted as a $96 \times 1$ matrix (one value for each slit), and the $P S F$ as a $288 \times 96$ matrix that, when matrix-multiplied by $D$, yields a $288 \times 1$ matrix (one value for each detector). Likewise, $\epsilon_{r e l}$ is a $288 \times 1$ matrix of relative efficiency values (one for each detector). This matrix is used to multiply each contribution elementwise to arrive at the total response. The values of the constants $A_{d}, A_{c p}$, and $A_{s s}$, which are part of the expressions for $D, P$, and $S$ as well as the exponent $\alpha$, were chosen to fit simulated data as described below.

Radiation transport simulations were performed for a ${ }^{244} \mathrm{Cm}$ line source placed at positions along the central axis of slit 49 at distances between 1 and $35 \mathrm{~cm}$ from the slit opening in $1 \mathrm{~cm}$ increments. Similar simulations were performed for a collimator having only slit 49 . The positions of the 49 th slit and the 35 points where the source is positioned are shown in Figure 13 for (left) a collimator with a single slit and (right) a collimator with 96 slits. 

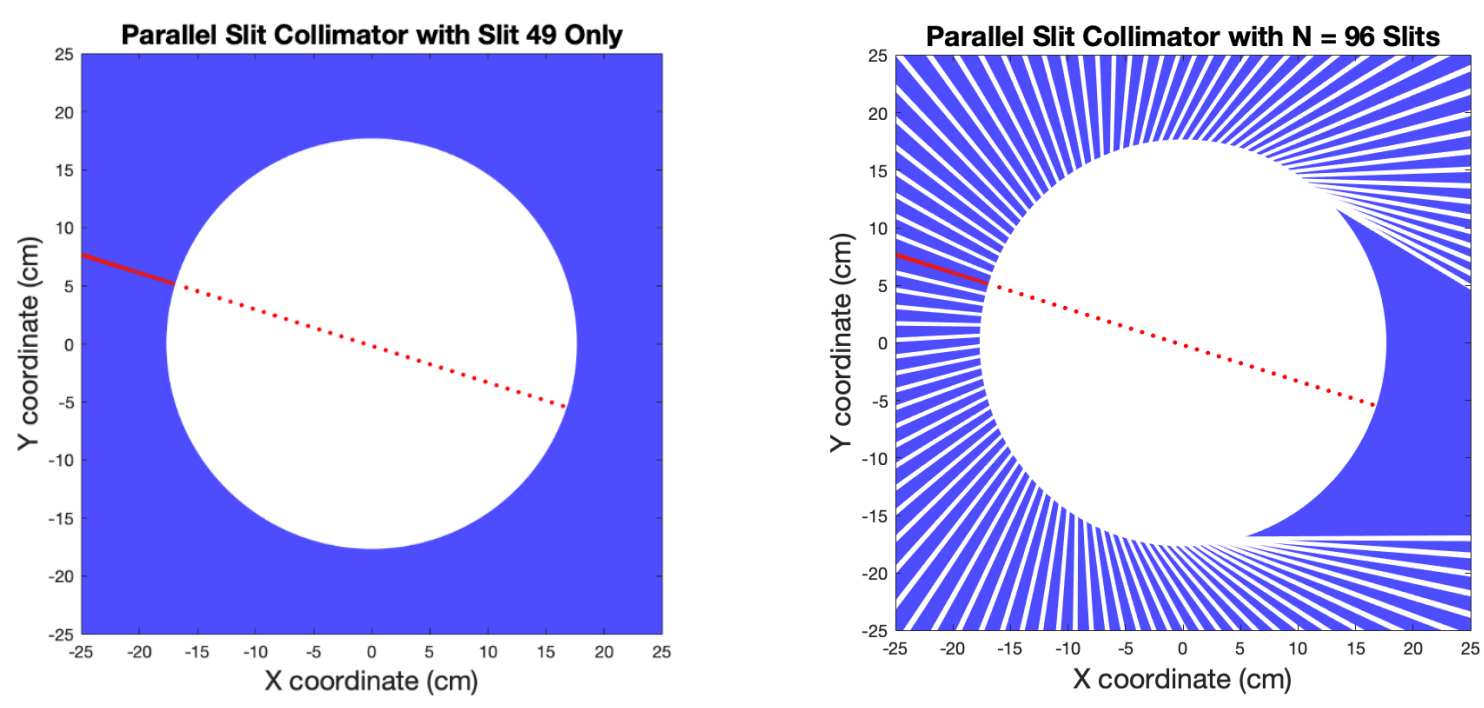

Figure 13. Positions of (red line) slit 49 and (red points) the 35 different source positions for (left) a collimator with a single slit and (right) a collimator with 96 slits.

The simulations of the collimator with the single slit were performed to generate data with a small contribution from collimator penetration. The results of the MCNP simulations for (left) the single collimator slit and (right) 96 collimator slits are shown on the upper row of Error! Reference source not found.. In these plots, the counts per source neutron are shown by the color scale for (y axis) each imager detector and ( $\mathrm{x}$ axis) the distance from the slit opening in centimeters. 

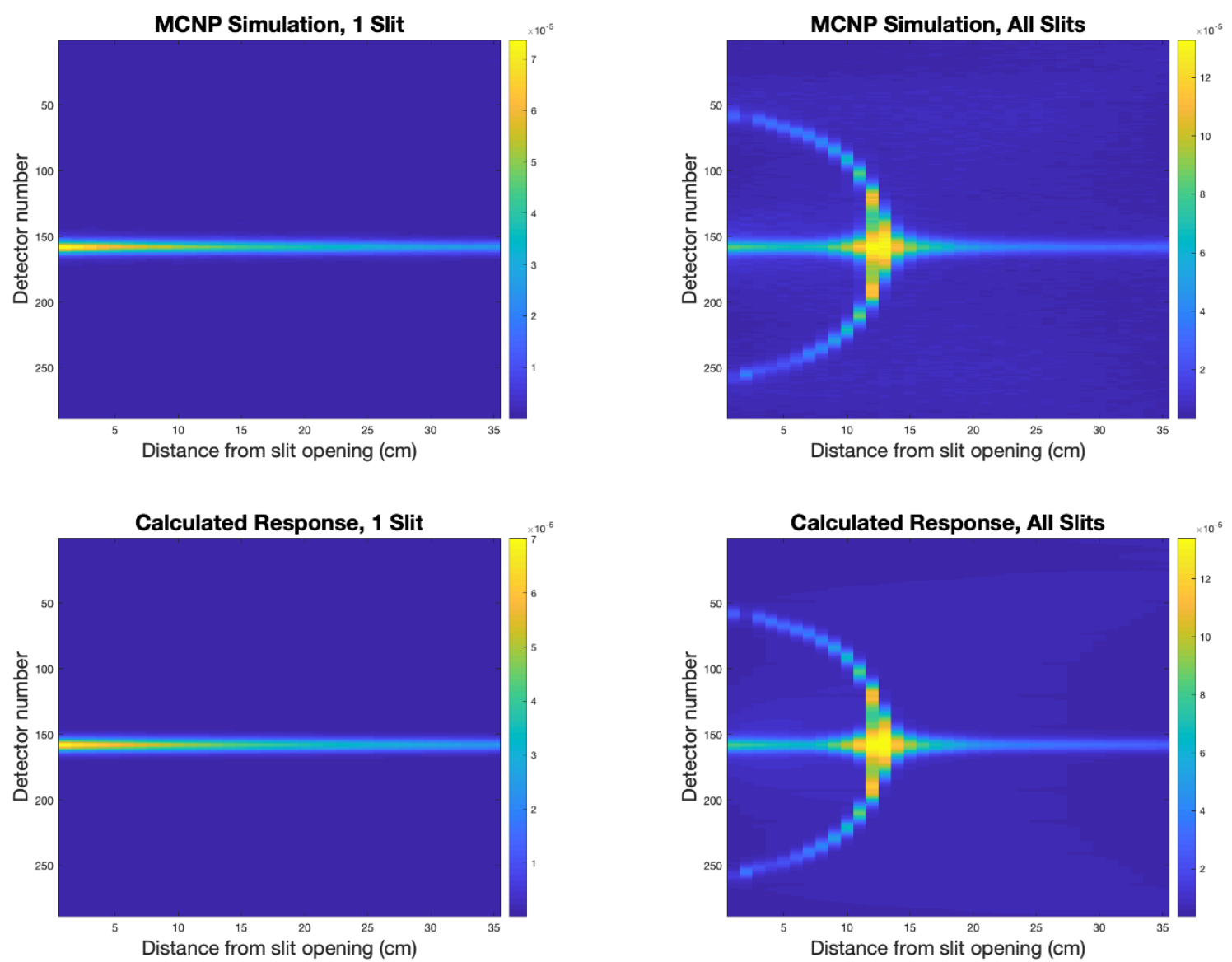

Figure 14. Comparison of (above) MCNP simulation and (below) analytical calculation of the imager response as a function of the source distance from the opening of slit 49 for (left) a collimator with only slit 49 and (right) the 96-slit collimator.

To match these data, a single set of constants $A_{d}$ and $A_{s s}$ were used to calculate all 35 distances for both collimators and separate values of $A_{c p}$ were used for each of the two collimators. Different values for the collimator penetration constant for the two collimators because the collimator with 95 additional slits is substantially more porous; in fact, the best-fit value of $A_{c p}$ for the single slit collimator is 0.053 times that of the 96-slit collimator. This process was repeated for several values of the exponent $\alpha$; the value of $\alpha=$ 1.43 produced a minimum in mean-squared error. For this value of $\alpha$, the best-fit value of $A_{d}=$ $3.390725 \times 10^{-4}, A_{s}=4.553 \times 10^{-6}$, and $A_{c p}=4.363601 \times 10^{-4}$.

Using these best-fit values, the calculated response for the (left) single slit collimator and (right) 96 slit collimator can be seen in the lower row of Error! Reference source not found.. Visually, the calculation matches the features of the MCNP simulation well. The comparison is also shown for individual source positions 5, 10, 15, 20, 25, and $30 \mathrm{~cm}$ from the slit opening in Figure 15 for the single-slit collimator and in Figure 16 for the 96-slit collimator. The analytical model achieves reasonable agreement with radiation transport simulation across the FOV for single slit or many slit collimators.

Note, one place for potential improvement of the multiple-slit collimator fit would be to improve the estimated collimator penetration for detectors numbered greater than 200 or less than 50 for points near the edge of the FOV. At present, the "local density" of the collimator is tabulated on a detector-bydetector basis as viewed by a source at the center of the FOV. The disagreement apparent for point 5 in 
Figure 16 is thought to originate from the apparent movement of the less dense regions with movement of the source in the FOV.
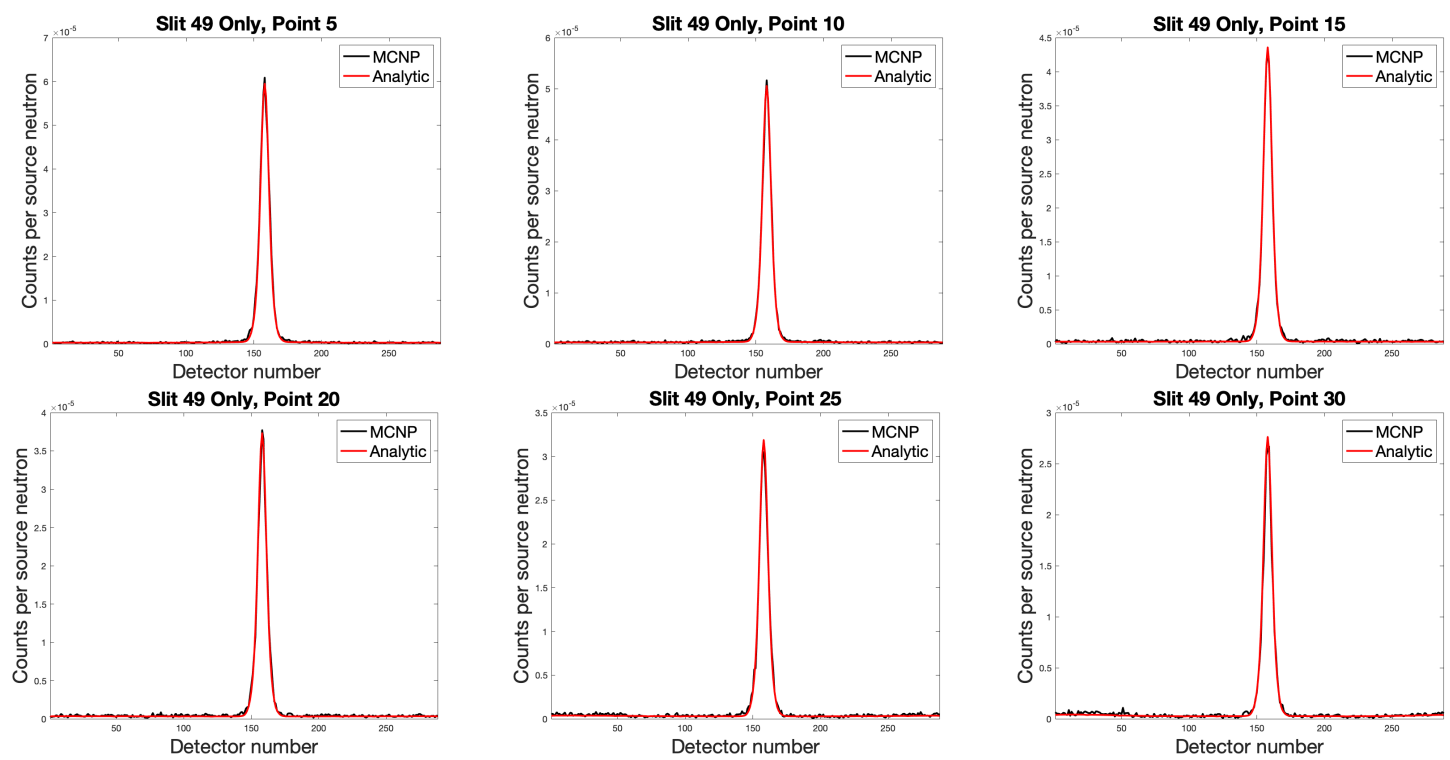

Figure 15. Simulated (black) and calculated (red) imager response for points 5, 10, 15, 20, 25, and $30 \mathrm{~cm}$ from the slit opening of a single-slit collimator where the slit is positioned in the same place as slit 49.
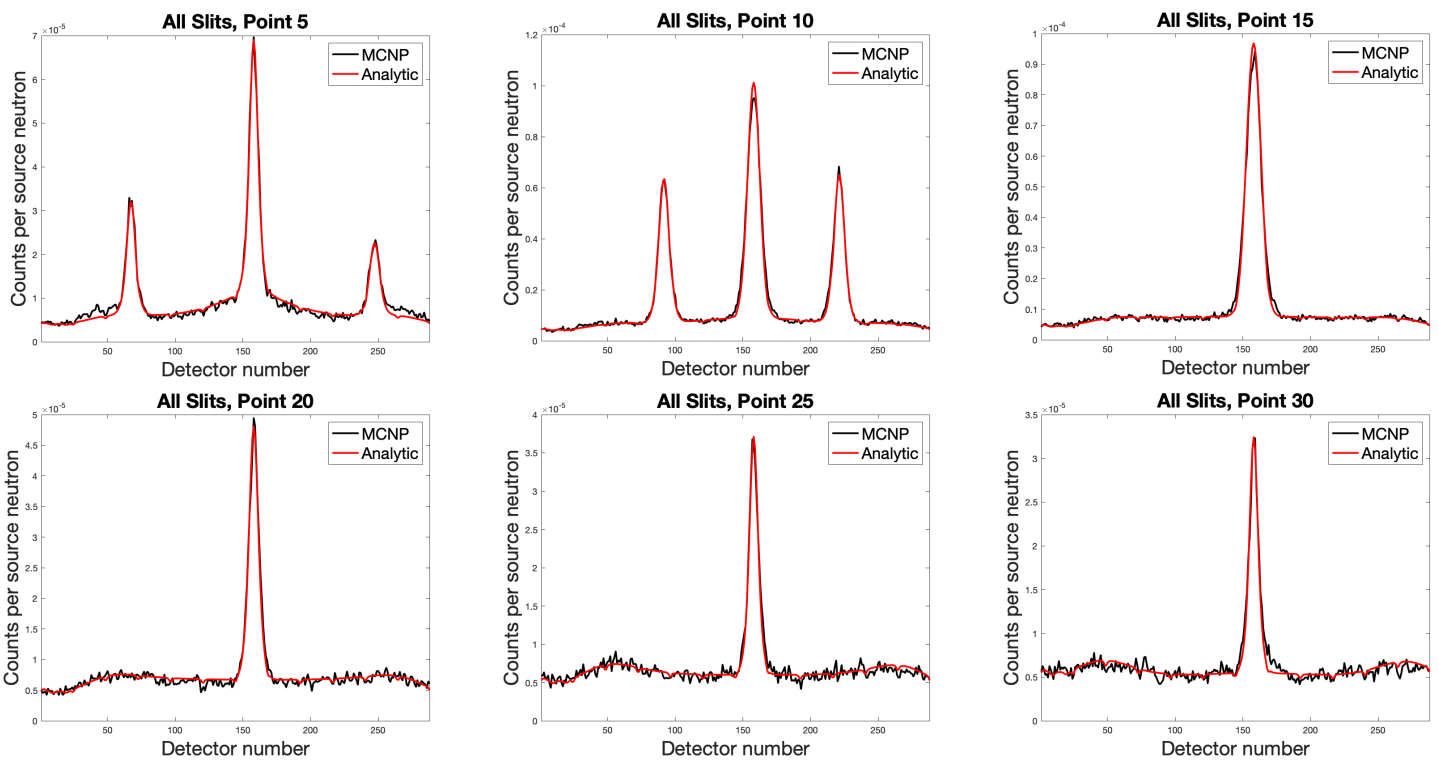

Figure 16. Simulated (black) and calculated (red) imager response for points 5, 10, 15, 20, 25, and $30 \mathrm{~cm}$ from the slit opening of slit 49.

With the relative contributions of the direct transmission, collimator penetration, and slit scatter set, the response of the imager can be calculated for any detector and angle of the collimator. An example calculated response is shown in Figure 17. This response is for detector 158 (corresponding to slit 49, marked in red) for the collimator orientation shown. Note, in addition to the primary line of response that aligns with slit 49 , there is also response from neighboring slits due to inter-detector scattering. 


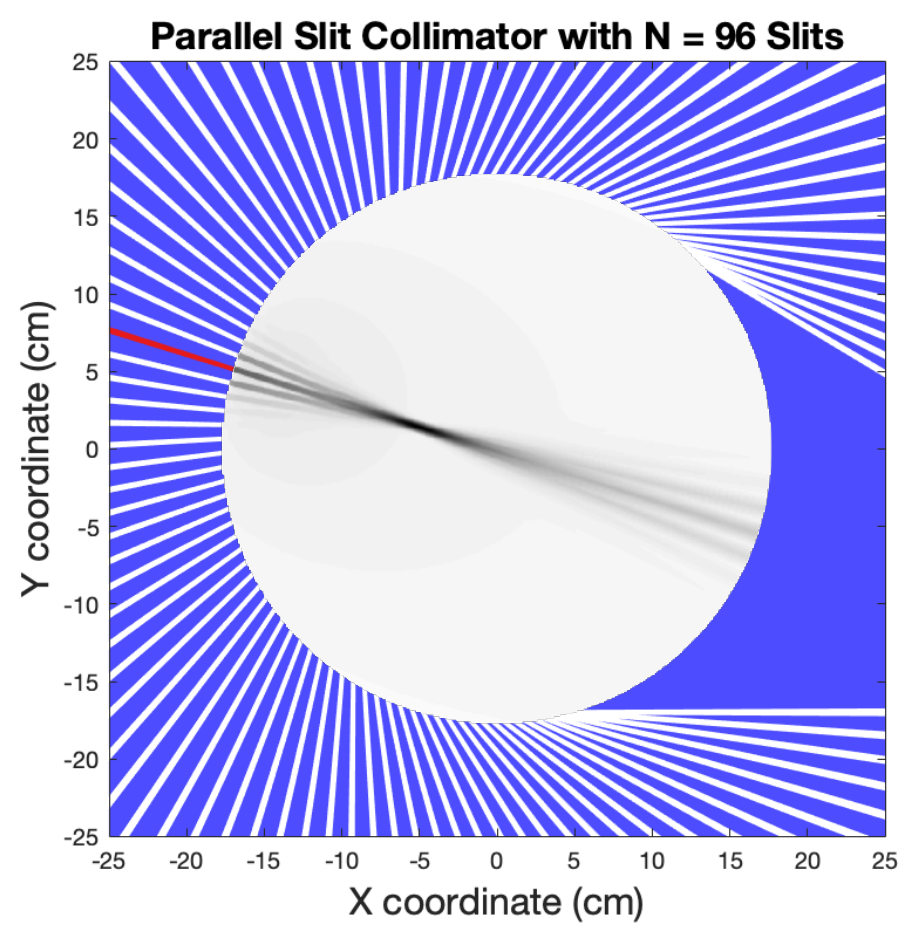

Figure 17. Schematic diagram of the collimator with example response shown for neutron detections in detector number 158.

\section{IMAGE RECONSTRUCTION}

The previous section concerned itself with computing the imager response across the FOV in millimeter increments for each detector and each orientation of the collimator. That response is necessary to perform iterative estimation of the distribution of the neutron activity in the FOV, or equivalently, to reconstruct a neutron emission image.

Suppose we have

- an image $\mathbf{x}$ whose pixel values $x_{j}$ correspond to the emitted neutron intensity for a voxel in space,

- $\quad$ measured projection data (i.e., a sinogram) $\mathbf{y}$ with values $y_{i}$ that correspond to detected counts for each detector and rotation of the collimator, and

- the imager response or system matrix $\boldsymbol{A}=\left[a_{i j}\right]$ such that $\boldsymbol{A} \boldsymbol{x}=\boldsymbol{y}$, or equivalently, $y_{i}=\sum_{j} a_{i j} x_{j}$.

Then, image reconstruction is the process of solving for $\boldsymbol{x}$ given $\boldsymbol{y}$ and $\boldsymbol{A}$. Generally speaking, $\boldsymbol{A}$ will be ill conditioned, making the explicit solution a poor choice because it will compound errors in $\boldsymbol{y}$. There are many methods to solve the problem iteratively, and all require the ability to "forward project" a guessed image to produce a trial sinogram.

Forward projection of a trial image to get a calculated sinogram is illustrated in Figure 18, where the values of one row of the system matrix $\boldsymbol{A}$ correspond to the probabilities connecting each voxel in the FOV to a particular detector pixel. Forward projection corresponds to summing the elementwise product of that probability map with the trial image. 

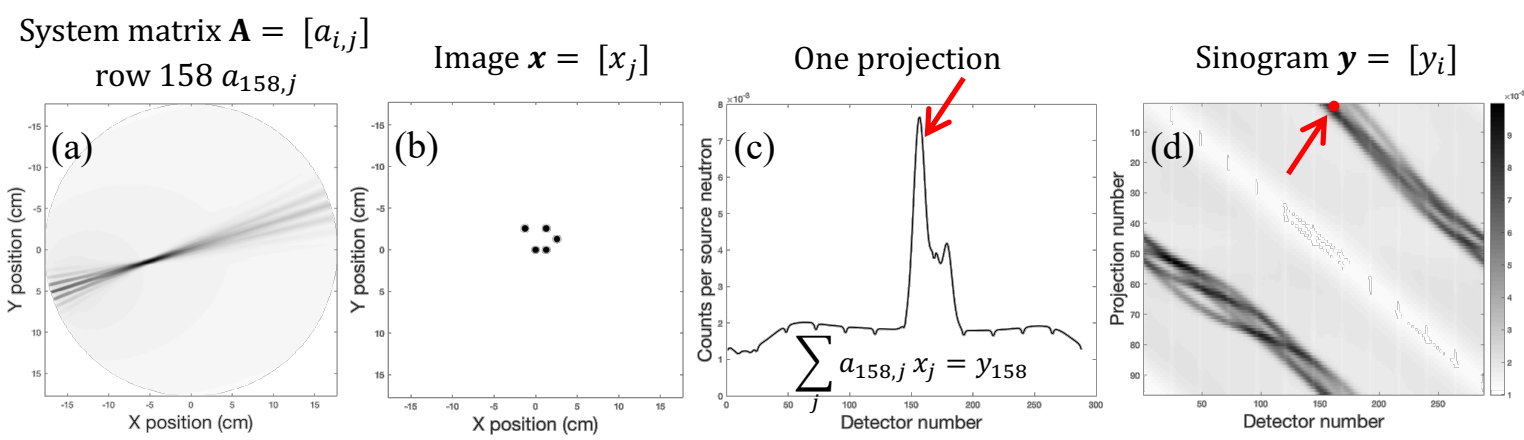

Figure 18. Illustration of forward projection to calculate a sinogram from an image. The values of one row of the system matrix multiply the image and sum to produce the counts per source neutron in a particular detector pixel and projection.

The scripts for performing image reconstruction are based on the maximum likelihood expectation maximization (MLEM) algorithm. That algorithm is derived from formally maximizing the log likelihoods, assuming the values $\boldsymbol{y}$ to be Poisson distributed about the solution $\boldsymbol{A x}$. This maximization results in an update equation for the $\mathrm{k}+1^{\text {st }}$ image values $x_{j}^{(k+1)}$ that is multiplicative.

$$
x_{j}^{(k+1)}=x_{j}^{(k)} \frac{1}{\sum_{i} a_{i j}} \sum_{i} a_{i j}\left(\frac{y_{i}}{\sum_{h} a_{i h} x_{h}^{(k)}}\right)
$$

In words, the $k^{\text {th }}$ trial image is forward projected to produce a trial sinogram. Then, the ratio of the measured-to-calculated sinograms is back projected to update the image by the weighted average of all the sinogram points connected to a particular image point. The derivation of the update equation is valid if the $y_{i}$ are Poisson distributed.

\subsection{RECONSTRUCTION OF SIMULATED MEASUREMENTS OF FIVE LINE SOURCES}

The first simulated tomographic measurement consisted of five line sources, each positioned in the center of one of the locations that would normally hold a fuel pin in a $17 \times 17$ fuel assembly. The line sources were placed so that pairs of line sources were at $1, \sqrt{2}$, and 2 times the closest possible spacing in the assembly. The five-source pattern is indicated in red in Figure 19.

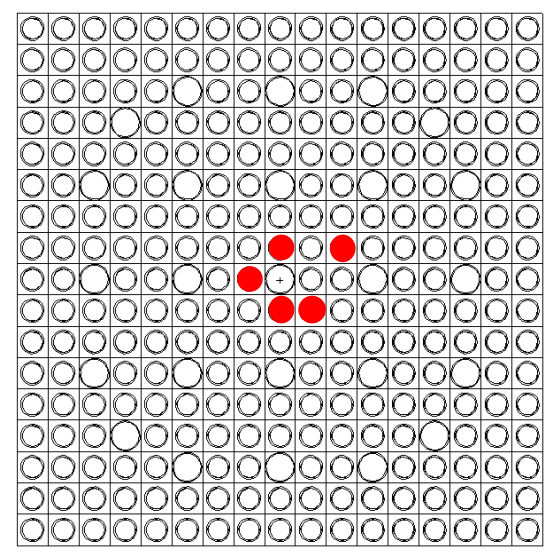

Figure 19. The positions of five line sources for the simulated tomographic imaging measurement are shown in red superimposed on a background of the layout of fuel pins in a $17 \times 17$ fuel assembly. 
For each projection, a total of $10^{8}$ source histories was simulated, corresponding to $2 \times 10^{7}$ emitted neutrons per line source. For a fuel assembly with a burnup of $45 \mathrm{GWd} / \mathrm{MTU}$, the emitted neutron intensity corresponds to approximately $3 \times 10^{5}$ neutrons per second per meter of fuel pin $(1 \mathrm{~m}$ is the length of fuel that will be in view of the imager). Therefore, the simulated number of neutrons corresponds to approximately 66 seconds per projection. A total of 96 projections were simulated for the tomographic measurement. Each of the projections corresponded to rotation of the collimator by the spacing of a single slit. The tomographic measurement with 96 projections would require about 106 minutes. The resulting neutron counts in each detector for each rotation angle is plotted on the left of Figure 20.
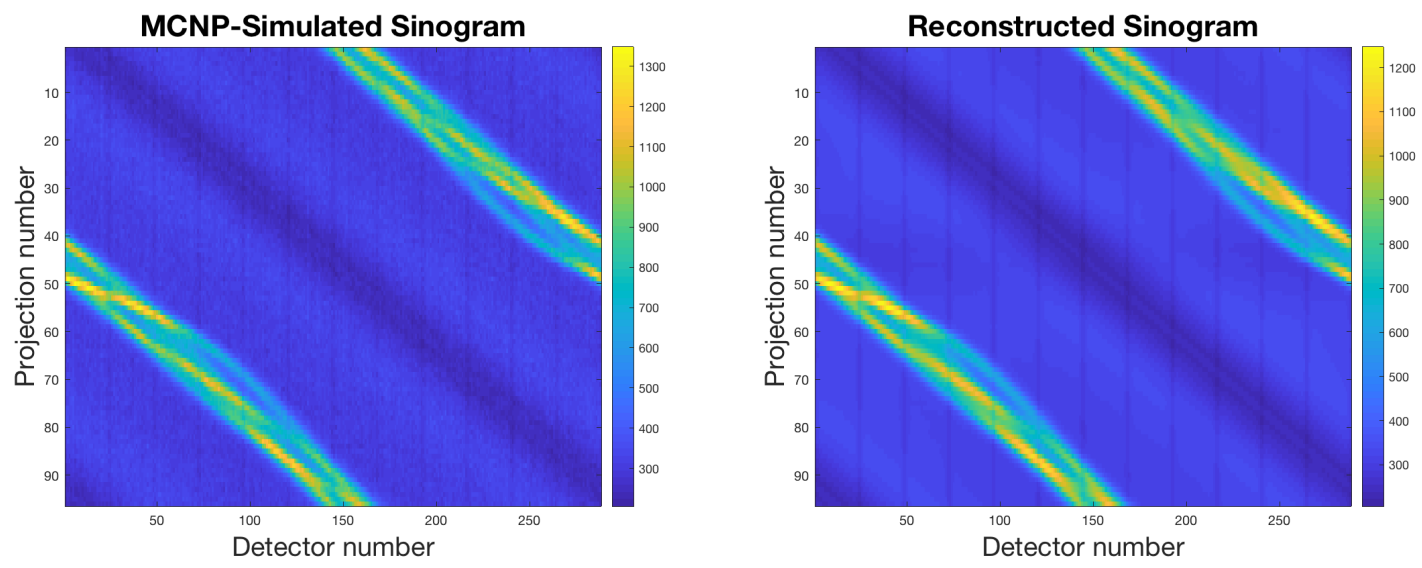

Figure 20. The (left) simulated and (right) reconstructed sinogram.

The data were reconstructed using the MLEM algorithm for 1,000 iterations. The resulting reconstructed sinogram is shown on the right of Figure 20. The reconstruction reproduces the visual features of the simulated data. To aid in comparison between the simulated measurement and reconstruction, comparison for projections 8 and 70 are shown in Figure 21. The reconstructed image that corresponds to the sinogram is shown in in Figure 22.
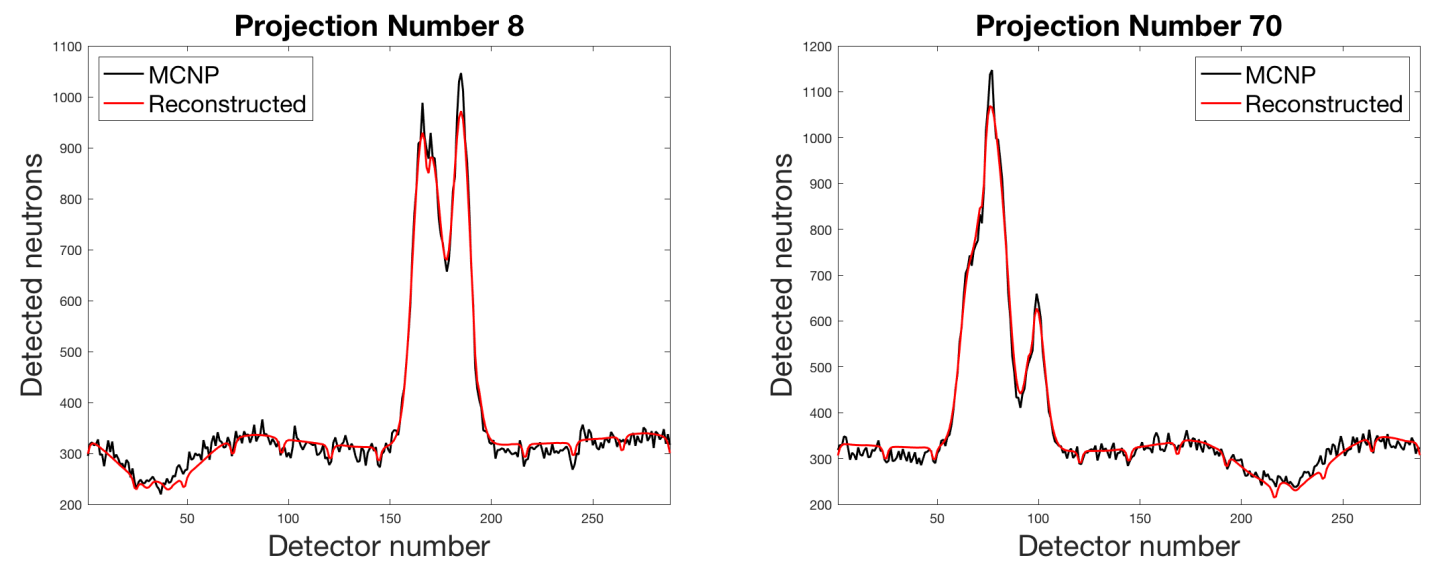

Figure 21. Comparison of MCNP simulation and MLEM reconstruction for (left) projection 8 and (right) projection 70. 


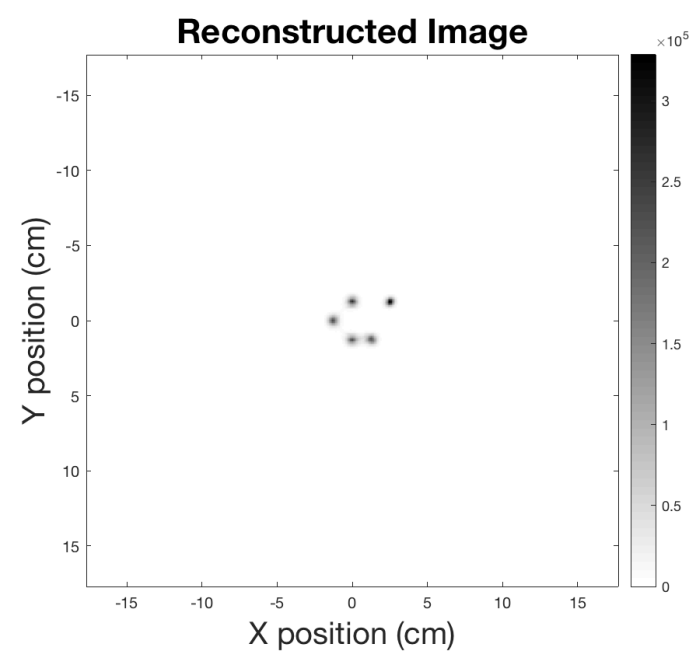

Figure 22. Image of five line sources reconstructed using the MLEM algorithm.

Each of the line sources is resolved from the others including the sources spaced at the closest distance from each other. This resolution can be examined as a function of the number of iterations. On the left of Figure 23, a transect through the two closest sources is shown as a function of the number of iterations. As iterations increase, the resolution improves, and more of the intensity is attributed to the source locations. On the right of Figure 23, the transect through the two closest sources is shown for 100, 200, and 1,000 iterations. Because the system matrix is not sparse, large numbers of iterations are needed to resolve sources close to one another.
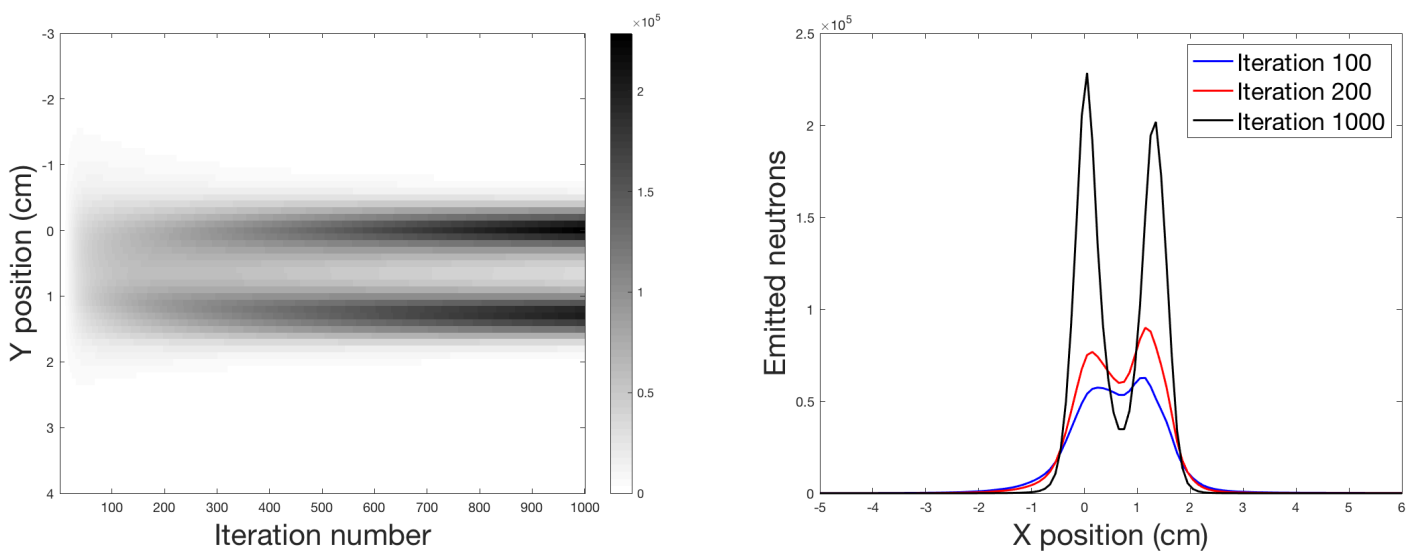

Figure 23. Comparison of the ability to resolve the closest sources with iteration number.

The transect through the closest two sources was fit to a pair of equal-width Gaussians for each iteration number. The resulting plot of full width at half maximum (FWHM) in millimeters as a function of iteration number is shown in Figure 24. For reference, the diameter of the neutron source component of a fuel pin is indicated by the dashed line just above $8 \mathrm{~mm}$. It takes more than 500 iterations to resolve line sources sufficiently to achieve a FWHM better than the size of a fuel pin. Note, sources better separated from their neighbors can be resolved in fewer iterations, as demonstrated by the red curve that shows the FWHM of a line source separated from its nearest neighbor by twice the fuel assembly array spacing. This result indicates that combined with iterative reconstruction the imager is expected to have sufficient spatial resolution to resolve individual fuel pins. 


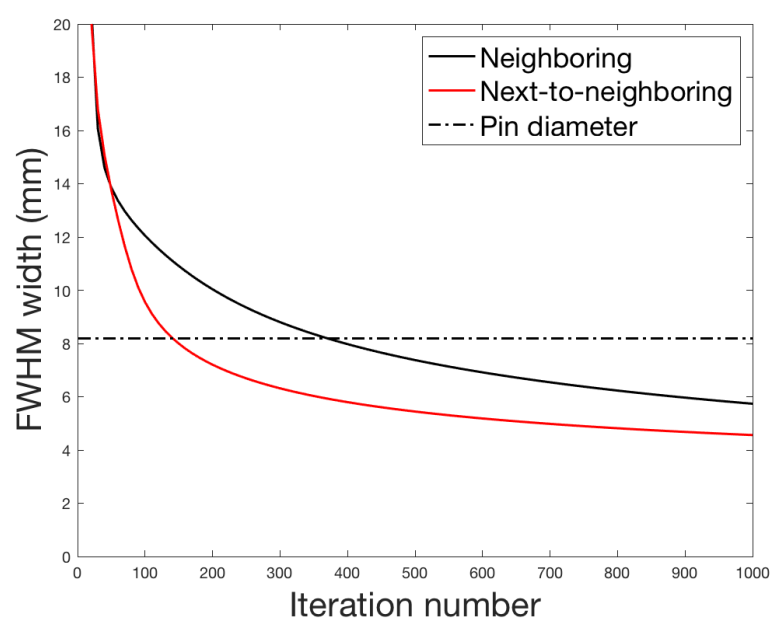

Figure 24. Resolution for line sources as a function of iteration for (black) neighboring sources and (red) next-to-neighboring sources.

\subsection{RECONSTRUCTION OF SIMULATED MEASUREMENT OF A FUEL ASSEMBLY}

The second simulated tomographic measurement consisted of a $17 \times 17$ fuel assembly with 265 fuel pins and 25 (empty) instrument channels. For each projection, a total of $10^{9}$ source histories was simulated. These neutrons were divided evenly among the active fuel pins, corresponding to $3.77 \times 10^{6}$ emitted neutrons per fuel pin. This simulated number of neutrons corresponds to approximately 12.6 seconds per projection for a fuel assembly with a burnup of $45 \mathrm{GWd} / \mathrm{MTU}$. A total of 96 projections were simulated for the tomographic measurement, and each of the projections corresponds to rotation of the collimator by the spacing of a single slit. A tomographic measurement with 96 projections would require about 20 minutes of measurement time. The source geometry for the $17 \times 17$ fuel assembly is shown in Figure 25 . The resulting simulated neutron counts in each detector for each rotation angle is plotted on the left of Figure 26.

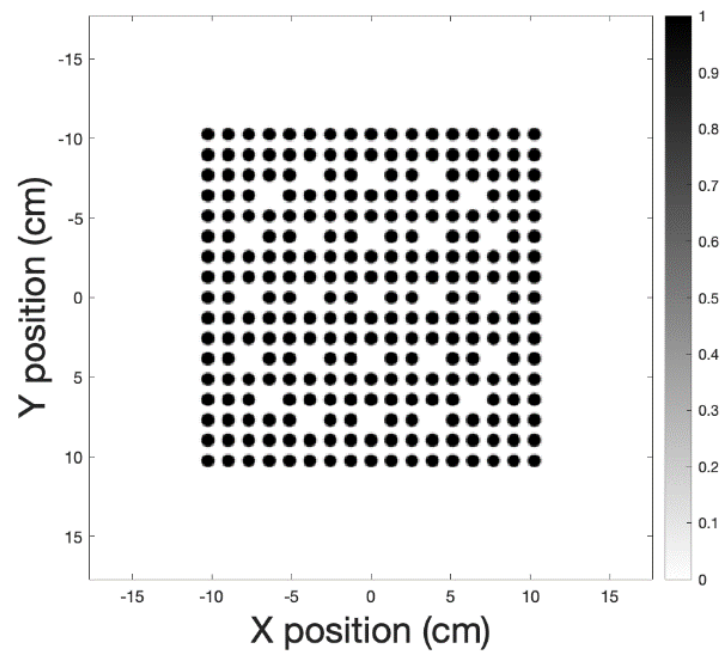

Figure 25. Schematic diagram of the source geometry for the simulated $17 \times 17$ fuel assembly. 

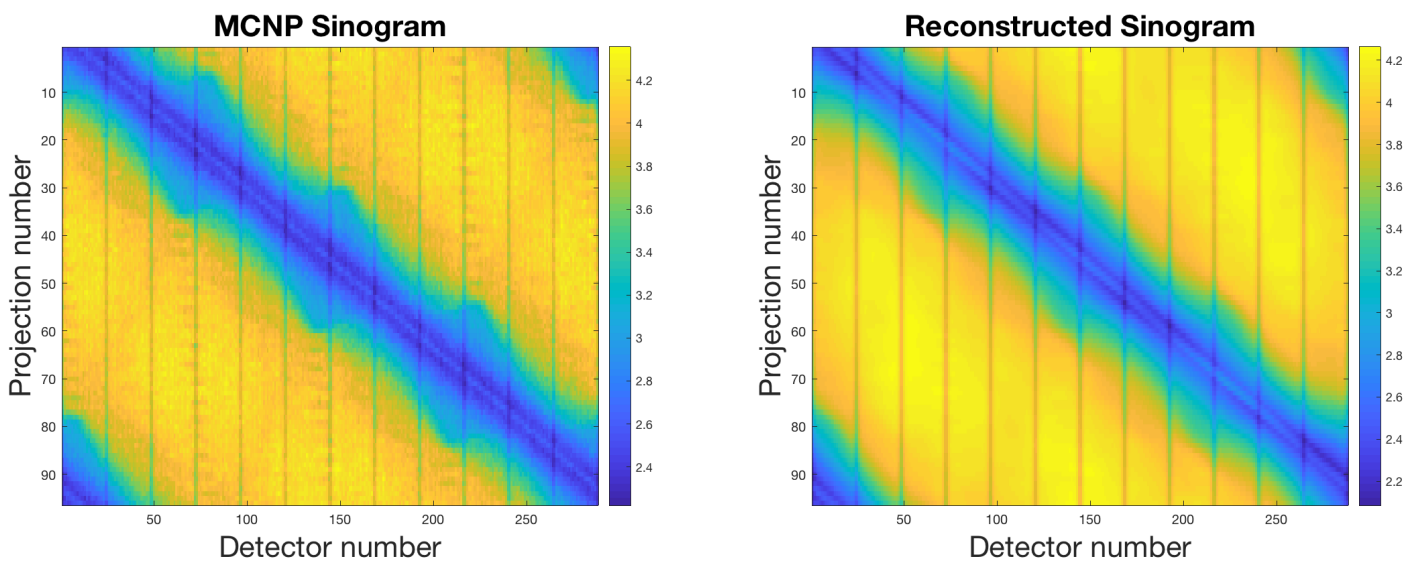

Figure 26. (Left) Simulated and (right) reconstructed sinograms for the $17 \times 17$ fuel assembly.

The authors recognize that in actual fuel, the source intensity from fuel pin to fuel pin would differ because of the position of the pin within the fuel assembly and its proximity to burnable poisons or instrument channels and the position of the fuel assembly within the reactor core. However, to generate data for initial image reconstructions, beginning with uniform pin-by-pin intensities throughout the assembly was preferable so that it would be easier to judge success.

As demonstrated for the reconstruction of five line sources, the imager response detailed in the previous section is sufficiently accurate to reconstruct images of a limited number of fuel pins in the center of the FOV. However, a full fuel assembly has 53 times more sources, so small systematic errors in the response can be comparable in intensity to a fuel pin. In addition, many of the fuel pins are near the edges of the FOV where scattering off the steel of the collimator is likely to be significant. At present, the accuracy of the imager response is insufficient to reconstruct faithful images of a full fuel assembly, but it still resolves many of the fuel pins. The authors are working to add an additional term to the response to account for scattering from the wall; however, this term requires additional development.

The simulated data were reconstructed using the MLEM algorithm for 1,000 iterations. The resulting reconstructed sinogram is shown on the right of Figure 26. The reconstruction reproduces many of the visual features of the simulated data but is not sufficiently converged to show fine detail. To aid in comparison between the simulated measurement and reconstruction, projection 56 is compared in Figure 27. Note, areas of discrepancy are highlighted by blue arrows. For this projection, the reconstruction underpredicts the response for the lowest 20 and highest 20 detectors and overpredicts the response near detectors 130 and 220. The reconstructed image that corresponds to the sinogram is shown in Figure 28. 


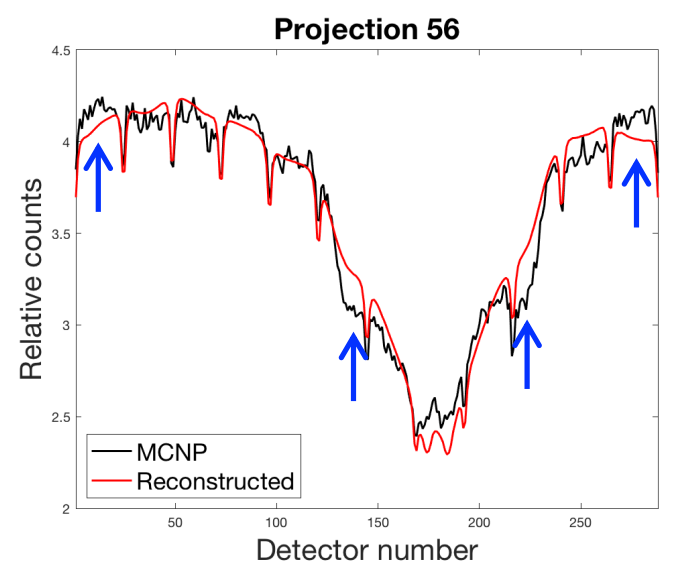

Figure 27. Comparison of MCNP-simulated and reconstructed sinograms for projection number 56. The blue arrows highlight areas of disagreement.

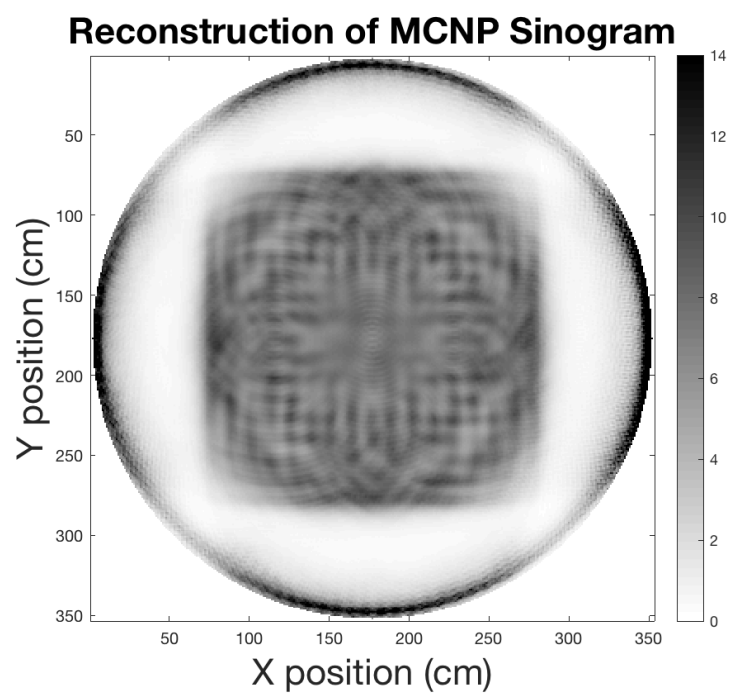

Figure 28. Reconstructed image of full fuel assembly. Reconstruction using the present response underrepresents the corners of the fuel assembly and attributes source strength to the edges of the FOV.

In the reconstructed image, many of the fuel pins are resolved. However, the fuel assembly is not uniformly illuminated, and in particular, the pins near the corners are essentially absent. This behavior of the reconstruction is unexpected because the corner pins are the closest and most readily observed without intervening material. In addition, a significant amount of neutron intensity is attributed to the edges of the FOV. Attribution of intensity to the edge of the FOV is a clear indication that the imager response is inadequate. However, it may accurately represent the collimator appearing to be a source of reflected neutrons. To investigate this notion, several additional analyses were performed.

To check whether there was an inherent problem with implementation of the reconstruction algorithm, an idealized sinogram was generated using the source geometry shown in Figure 25 and the forward projection of the imager. This way, image reconstruction could be performed on a data set in which the system model used by the reconstruction was known to be correct by construction. The reconstructed image using this data set is shown in Figure 29. For this image, individual fuel pins are visible across the fuel assembly. That is, the image reconstruction algorithm works sufficiently well provided the imager response is sufficiently accurate (and the data has sufficient statistical precision). 


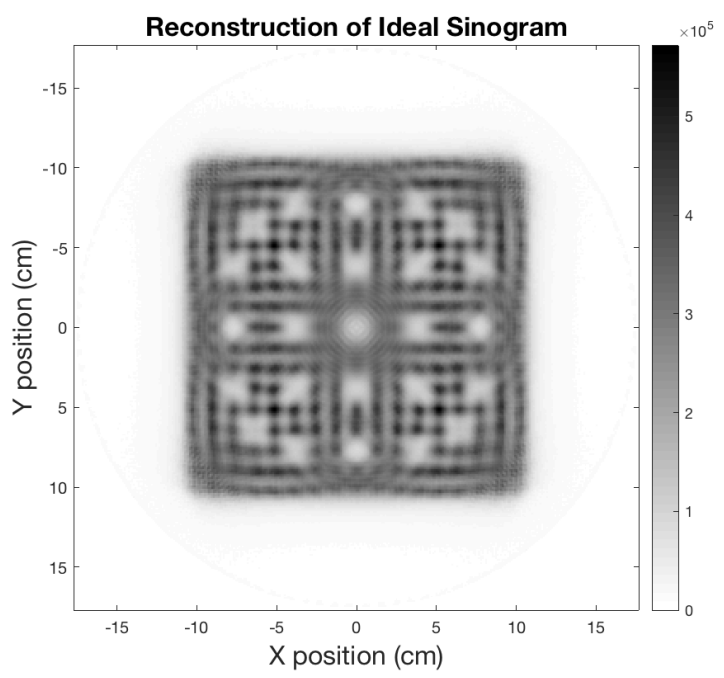

Figure 29. The reconstructed image of the idealized sinogram (calculated using the imager response). Reconstruction using the same response that generates the sinogram correctly reconstructs the corners of the fuel assembly.

Additional tests were performed to elucidate causes of inaccuracy in the reconstruction of the MCNPsimulated tomographic measurement of the full fuel assembly. In particular, image reconstruction was performed where attribution of neutron intensity was limited to single values at the locations of fuel pins. This process eliminates the ability to reconstruct intensity at the edges of the FOV and reduces the number of unknowns from approximately 100,000 pixels to the value of 265 fuel pins. To accomplish this, the expected sinogram was calculated for each active fuel pin position. For example, pin positions 1 , 144, and 179 are shown with their associated calculated sinograms in Figure 30. Then, the forwardprojected sinogram consists of the sum of the 265 sinograms weighted by their neutron intensity. The reconstructed image using this methodology is shown in Figure 31 (left) as a $17 \times 17$ array of values and (right) as an idealized image with each fuel pin shaded by its reconstructed neutron intensity. Note, the fuel assembly has more uniform values but has additional attributed neutron intensity at the edges. The resulting reconstructed sinogram is shown on the right of Figure 32. To aid in comparison between the simulated measurement and reconstruction, comparison of projection 56 is shown in Figure 33. 

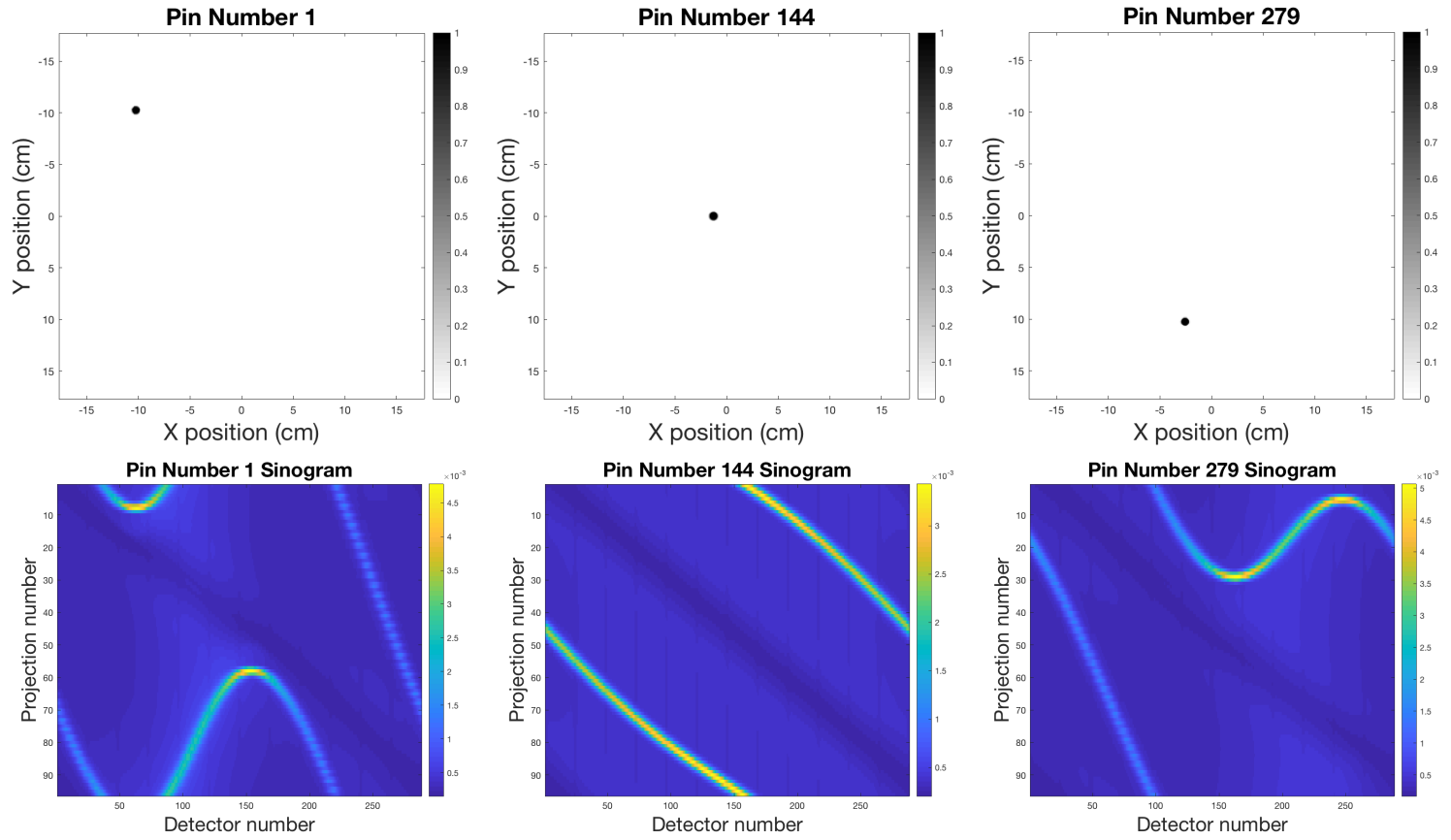

Figure 30. Position and sinogram for three example fuel pin positions.
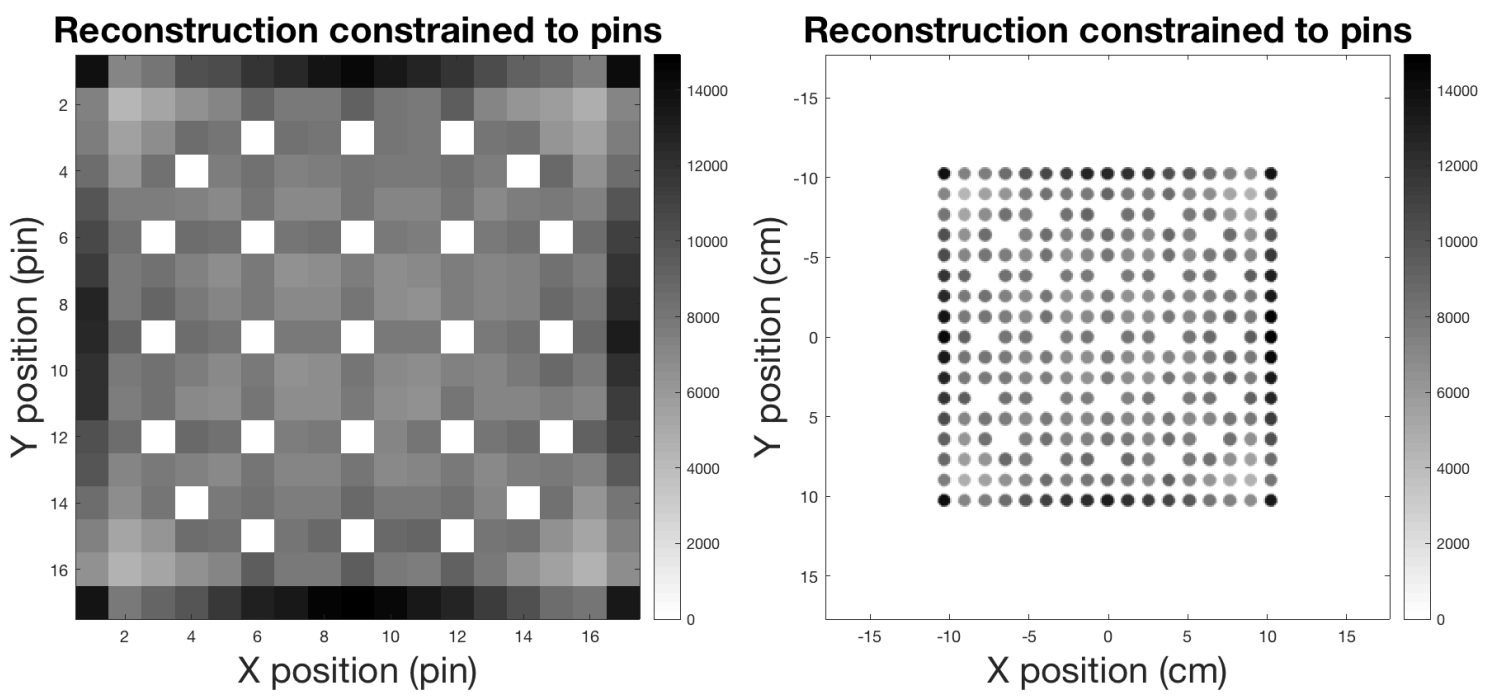

Figure 31. Reconstructed neutron source values for fuel pins (left) shown as a $17 \times 17$ array and (right) as an idealized image with the fuel pins shaded by the appropriate value. 

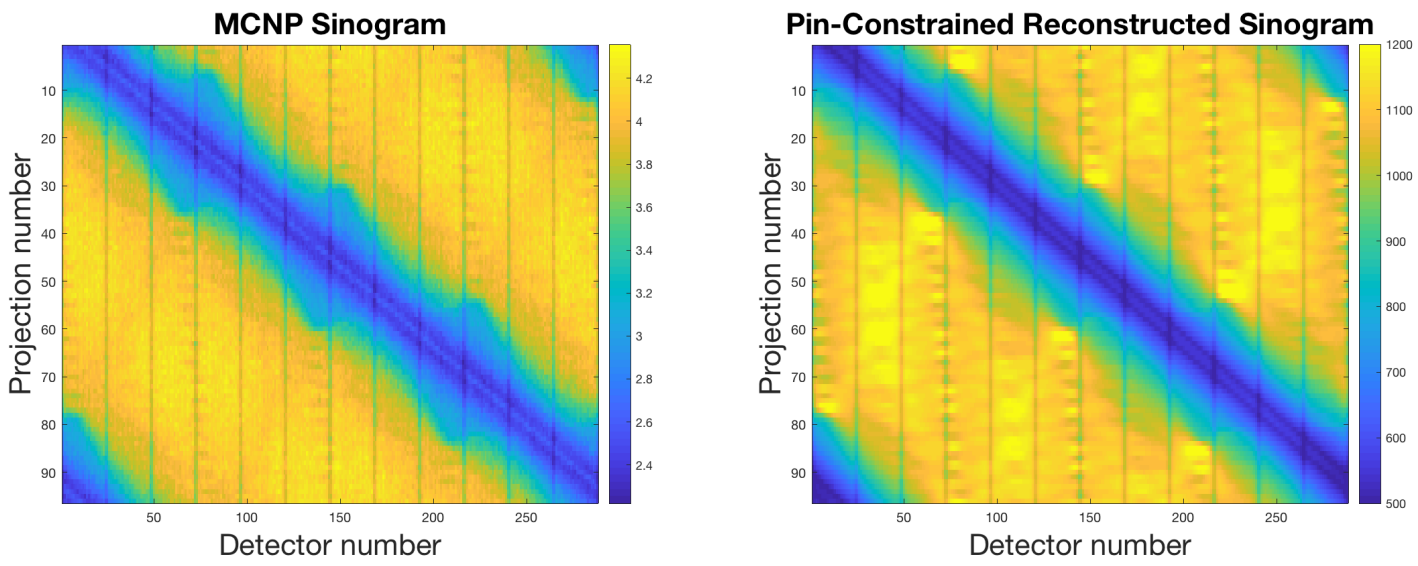

Figure 32. (Left) Simulated and (right) reconstructed sinogram.

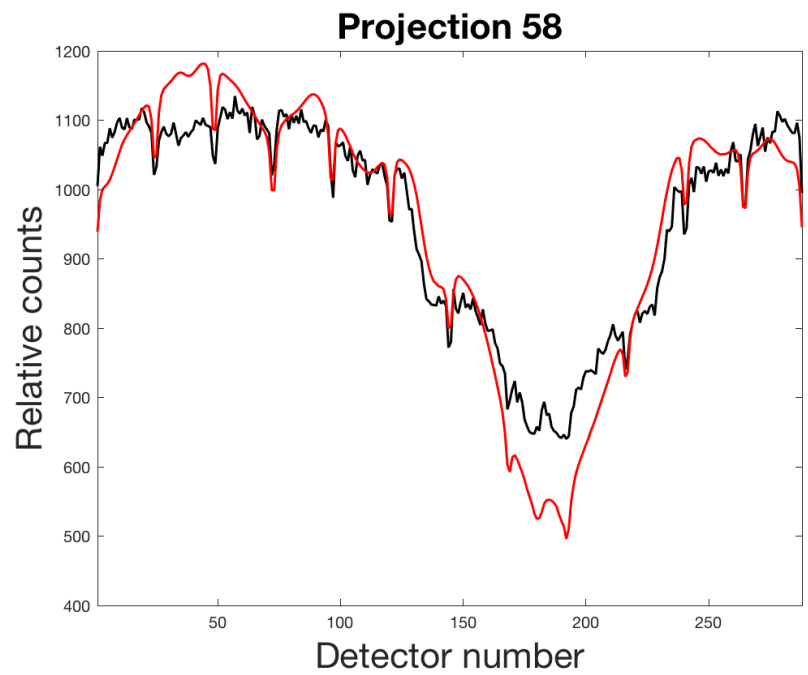

Figure 33. Comparison of MCNP-simulated and pin-constrained reconstructed sinograms for projection number 56.

\section{SUMMARY AND CONCLUSIONS}

The present work continues development of the parallel-slit ring collimator for passive neutron emission imaging of spent fuel. A major thrust of this effort was development of an analytical description of the imager response that can be used to calculate the system matrix for all points in the FOV, all detectors, and all orientations of the collimator with modest computing resources.

The novel parallel-slit ring collimator design simultaneously solves the principal challenges to tomographic neutron measurements of spent fuel, including effectively collimating neutrons, achieving sufficient spatial resolution, maintaining sufficient efficiency for the modest neutron source strength from spent fuel, and maintaining sufficient insensitivity to the gamma-ray flux to allow meaningful neutron measurements. However, the collimator design also complicates the neutron response. In particular, the neutron radiation that penetrates the collimator is a significant fraction of the true signal and spatially dependent. As a result, inaccuracy in accounting for the intensity and spatial dependence of radiation that penetrates the collimator can result in artifacts with similar intensity to fuel pins in reconstructed images. To accurately reconstruct images, the imager response is required to be highly accurate across the FOV. 
The present analytic imager response reproduces the response of individual sources across the FOV with sufficient accuracy to reconstruct small numbers of sources near the center of the FOV. Using this response, tomographic image reconstruction with sufficient spatial resolution to resolve individual fuel pins in a fuel assembly has been demonstrated for simulated imaging measurements. Unfortunately, the present response is not sufficiently accurate to faithfully reconstruct full fuel assemblies. This limitation is believed to arise from inaccuracy in the imager response that originates from scattering off the inner diameter of the collimator surrounding the imager FOV. For a few sources, the contribution of this term can be neglected because its intensity is a small fraction of the individual sources. However, with 50 times more sources, its intensity is 50 times more while the intensity of the individual fuel pins does not increase. At present, additional simulations are underway to account for and include this term in the imager response.

Because faithful image reconstruction has not yet been demonstrated for full fuel assemblies, it is premature to predict the expected magnitude of observable defects in spent fuel.

\section{ACKNOWLEDGEMENTS}

This work is supported by the support of the US Department of Energy, Office of Defense Nuclear Nonproliferation Research and Development in the National Nuclear Security Administration (NA-22). Additional contributions to this research were performed under appointment to the Nuclear Nonproliferation International Safeguards Fellowship Program sponsored by the National Nuclear Security Administration's Office of International Nuclear Safeguards (NA-241).

\section{REFERENCES}

[1] T. Honkamaa et al., "A Prototype for Passive Gamma Emission Tomography,” International Atomic Energy Agency Safeguards Symposium, 2014.

[2] M. Mayorov et al., "Gamma Emission Tomography for the Inspection of Spent Nuclear Fuel," 2017 IEEE Nuclear Science Symposium and Medical Imaging Conference (NSS/MIC), Atlanta, GA, 2017, pp. 1-2.

[3] P. A. Hausladen, M. A. Blackston, and R. J. Newby, "Demonstration of Emitted-neutron Computed Tomography to Quantify Nuclear Materials,” Report no. ORNL/TM 357, Oak Ridge National Laboratory, Oak Ridge, TN, 2011.

[4] P. A. Hausladen, A. S. Iyengar, L. Fabris, J. Yang, and J. Hu, "A Design Study of the Parallel-Slit Ring Collimator for Fast Neutron Emission Tomography of Spent Fuel," Report no. ORNL/SPR2018/975, Oak Ridge National Laboratory, Oak Ridge, TN, 2018. 